\title{
A study of accretion and disk diagnostics in the NGC 2264 cluster $^{\star}, \star \star$
}

\author{
Alana P. Sousa ${ }^{1,2}$, Silvia H. P. Alencar ${ }^{1}$, Luisa M. Rebull ${ }^{3}$, Catherine C. Espaillat ${ }^{4}$, \\ Nuria Calvet $^{5}$, and Paula S. Teixeira ${ }^{6}$ \\ 1 Departamento de Física-Icex-UFMG, Antônio Carlos 6627, 31270-901 Belo Horizonte, MG, Brazil \\ e-mail: alana@fisica.ufmg.br \\ 2 Université Grenoble Alpes, IPAG, 38000 Grenoble, France \\ 3 Infrared Science Archive (IRSA), IPAC, California Institute of Technology, 1200 E. California Blvd., Pasadena, CA 91125, USA \\ ${ }^{4}$ Department of Astronomy, Boston University, 725 Commonwealth Avenue, Boston, MA 02215, USA \\ 5 Department of Astronomy, University of Michigan, 830 Dennison Building, 500 Church Street, Ann Arbor, MI 48109, USA \\ 6 Scottish Universities Physics Alliance (SUPA), School of Physics and Astronomy, University of St. Andrews, North Haugh, Fife, \\ KY16 9SS St. Andrews, UK
}

Received 27 March 2019 / Accepted 1 August 2019

\begin{abstract}
Context. Understanding disk dissipation is essential for studying how planets form. Disk gaps and holes, which almost correspond to dust-free regions, are inferred from infrared observations of T Tauri stars (TTS), indicating the existence of a transitional phase between thick accreting disks and debris disks. Transition disks are usually referred to as candidates for newly formed planets.

Aims. We searched for transition disk candidates belonging to NGC 2264. Using stellar and disk parameters obtained in the observational multiwavelength campaign CSI 2264, we characterized accretion, disk, and stellar properties of transition disk candidates and compared them to systems with a full disk and diskless stars.

Methods. We modeled the spectral energy distribution (SED) of a sample of 401 TTS, observed with both CFHT equipped with MegaCam and IRAC instrument on the Spitzer, with Hyperion SED fitting code using photometric data from the U band $(0.3 \mu \mathrm{m})$ to the Spitzer/MIPS $24 \mu \mathrm{m}$ band. We used the SED modeling to distinguish transition disk candidates, full disk systems, and diskless stars.

Results. We classified $\sim 52 \%$ of the sample as full disk systems, $\sim 41 \%$ as diskless stars, and $\sim 7 \%$ of the systems as transition disk candidates, among which seven systems are new transition disk candidates belonging to the NGC 2264 cluster. The sample of transition disk candidates present dust in the inner disk similar to anemic disks, according to the $\alpha_{\text {IRAC }}$ classification, which shows that anemic disk systems can be candidate transition disks. We show that the presence of a dust hole in the inner disk does not stop the accretion process since $82 \%$ of transition disk candidates accrete and show $\mathrm{H} \alpha$, UV excess, and mass accretion rates at the same level as full disk systems. We estimate the inner hole sizes, ranging from 0.1 to $78 \mathrm{AU}$, for the sample of transition disk candidates. In only $\sim 18 \%$ of the transition disk candidates, the hole size could be explained by X-ray photoevaporation from stellar radiation.
\end{abstract}

Key words. stars: pre-main sequence - stars: variables: T Tauri, Herbig Ae/Be - accretion, accretion disks - protoplanetary disks

\section{Introduction}

Circumstellar disks are ubiquitous around young stars and are the sites of planet formation. The analysis of disk structure and evolution is therefore an essential step in understanding the formation of planets. There are still many questions as to how the gas and dust in circumstellar disks evolve into planetary systems. Infrared (IR) observations of young low mass stars show that the number of circumstellar disks decreases with age with a typical timescale for dissipation around $\sim 6 \mathrm{Myr}$ (e.g., Haisch et al. 2001; Hernández et al. 2007; Ribas et al. 2014).

Several mechanisms of disk dissipation have been proposed in the literature. The inner disk is partly dissipated by accretion onto the star with a typical rate of about $10^{-8} M_{\odot} \mathrm{yr}^{-1}$ (Hartmann

\footnotetext{
* Full Tables 2 and 3 are only available at the CDS via anonymous ftp to cdsarc.u-strasbg. fr (130.79.128.5) or via http://cdsarc. u-strasbg.fr/viz-bin/qcat?J/A+A/629/A67

$\star \star$ This work is based on observations made with FLAMES instrument on the Very Large Telescope under program ID 088.C-0239.
}

et al. 1998) in the T Tauri phase. Disks can be photoevaporated by the central star's high-energy radiation, which is a very efficient mechanism for gas dispersal, once the mass accretion rate drops below the photoevaporation mass loss rate (Clarke et al. 2001; Adams et al. 2004; Owen et al. 2011; Rosotti et al. 2013; Alexander et al. 2014). The disk material may be driven out of the system through disk winds and jets (Konigl 1989; Pelletier \& Pudritz 1992; Safier 1993; Shu et al. 1994). The disk can also be consumed in the coagulation of grains and planet formation (Papaloizou \& Terquem 1999; Dullemond \& Dominik 2005; Hollenbach et al. 2005; Zhu et al. 2011).

Circumstellar disks are often detected due to IR excess with respect to the stellar photosphere. Dust in the disk is heated by the central star and accretion and it then reemits IR wavelengths. The spectral energy distribution (SED) of a star with an optically thick disk, therefore, presents an IR excess added to the stellar photospheric contribution. As the disk disperses, the SED gradually shows less IR excess (Lada 1987). Furthermore, regions of the disk that are almost dust-free, that correspond to 
gaps and holes, are inferred from IR observations of T Tauri stars (TTS). Inner disk hole SEDs are characterized by a lack of emission excess in the near-IR but normal thick disk IR emission at mid-IR wavelengths (e.g., Koepferl et al. 2013; Espaillat et al. 2014). Inner disk clearing was confirmed with resolved millimeter continuum images (Hughes et al. 2009; Andrews et al. 2011). ALMA later revealed inner disk substructures such as rings and thin gaps less than $1 \mathrm{AU}$ wide (e.g., Andrews et al. 2016, 2018).

The formation of holes or gaps in the disk can be explained by dust coagulation due to Brownian motion of dust grains (Ormel et al. 2007; Garaud et al. 2013), or the presence of a newly formed planet that dynamically clears a specific region of the disk (Calvet et al. 2002; Zhu et al. 2011; Owen 2016). Photoevaporation may also clear a hole in the circumstellar disk but only in the final stages of disk accretion (Owen et al. 2011). Most systems with an inner disk hole, however, still accrete at typical T Tauri rates (e.g., Merín et al. 2010; Espaillat et al. 2012; Manara et al. 2014). These disks are called transition disks. Transition disks are not common in star forming regions, representing only about $10 \%$ of young star-disk systems (Owen 2016; Ansdell et al. 2016). This indicates that disk dispersal is rapid compared to disk lifetime.

We searched for transition disk candidates in the young ( $3 \mathrm{Myr}$ ) stellar cluster NGC 2264, located at a distance of $\sim 760 \mathrm{pc}$ from the Sun. We used data from the Coordinated Synoptic Investigation of NGC 2264 (CSI 2264) observational campaign (Cody et al. 2013) to characterize the transition disk candidates in terms of various accretion diagnostics ( $\mathrm{H} \alpha$ line emission, ultraviolet excess), disk parameters (IR excess), and stellar parameters (mass, effective temperature, radius) by comparing them to the characteristics of systems with full disks and diskless stars.

In Sect. 2 we describe the data used in this work, in Sect. 3 we explain the sample selection criteria, and in Sect. 4 we present the determination of accretion and disk characteristics of our sample. The discussion and analysis of inner hole properties are in Sect. 5, and in Sect. 6 we present our conclusions.

\section{Observations}

We used data from the CSI 2264, an international campaign that included simultaneous photometric and spectroscopic observations of the young cluster NGC 2264 with satellites and groundbased telescopes at various wavelengths (see Cody et al. 2013, for more details about the campaign). During the CSI 2264 campaign, NGC 2264 was simultaneously observed in the optical with the CoRoT satellite for 40 days and with Spitzer satellite (Werner et al. 2004) at 3.6 and $4.5 \mu \mathrm{m}$ for 30 days. We obtained 20 epochs of observations on the Very Large Telescope (VLT) equipped with the Fiber Large Array Multi Element Spectrograph (FLAMES), four to six observations simultaneously with the CoRoT data, depending on the target. Photometry in the $u$ and $r$ bands was also obtained with MegaCam (Canada-FranceHawaii Telescope-CFHT) for 14 nights about one month after the end of the CoRoT observations.

Additionally to the CSI 2264 data, to construct SEDs in Sect. 4.2, we also used data from catalog surveys, such as $U B V R_{c} I_{c}$ optical photometry from Rebull et al. (2002), ugriz optical photometry from SDSS (Gunn et al. 1998), near-IR photometry $J H K_{s}$ from 2MASS, IRAC (Fazio et al. 2004), and MIPS (Rieke et al. 2004) data from Spitzer satellite, and observations from the Wide-field Infrared Survey Explorer (WISE) at 3.4, 4.6, 12.0, and $22 \mu \mathrm{m}$ (Wright et al. 2010).

\section{Sample of stars}

There is no consensus in the literature on how to classify a stardisk system as a transition disk. In the simplest definition, transition disks are systems with little near-IR excess and strong midIR excess. In Sect. 4.2, we discuss some different ways from the literature to define a transition disk.

We selected inner disks with holes and gaps based on the flux observed in the near and mid-IR, and on the fitting of SEDs, that is, the flux emitted by the sources in various photometric bands vs. their central wavelengths. As we intend to analyze the accretion and disk properties of transition disk systems, our sample of stars only contains TTS that were observed with IRAC instrument on the Spitzer, which had an $\alpha_{\text {IRAC }}$ index (the slope of the SED between $3.6 \mu \mathrm{m}$ and $8 \mu \mathrm{m}$ ) measured by Teixeira et al. (2012) and were also observed in $u, g, r$, and $i$ bands at CFHT with MegaCam (Venuti et al. 2014). The IRAC completeness limits, from Teixeira et al. (2012), are $13.25 \mathrm{mag}$ for the $3.6 \mu \mathrm{m}, 4.5 \mu \mathrm{m}$, and $5.8 \mu \mathrm{m}$ IRAC bands and $12.75 \mathrm{mag}$ for the $8 \mu \mathrm{m}$ IRAC band. The IRAC sample from Teixeira et al. (2012) was also limited to magnitude uncertainties smaller than $0.1 \mathrm{mag}$ for all IRAC bands. Consequently, some IRAC sources belonging to NGC 2264 could be missing compared to other literature works that used IRAC observations. Our sample is composed of 401 TTS that were observed with both MegaCam and IRAC and appeared in Teixeira et al. (2012), to select the systems with the largest number of measured stellar, accretion, and disk parameters. As the sample is not complete, disk frequencies inferred from these data are not reliable.

We calculated synthetic SEDs for all the objects in our sample, using the Python-based fitting code sedfitter ${ }^{1}$ (Robitaille 2017) based on Hyperion, an open-source parallelized threedimensional dust continuum radiative transfer code by Robitaille (2011). This fitting model consists of modular sets with different components that can include a stellar photosphere, a disk, an envelope, and ambipolar cavities. The code allows the user to choose the best set of models for their sample of stars and eliminate unphysical models. We tested three sets of models ${ }^{2}$ : model 1 - only a central star; model 2 - a central star and a passive disk, which is a disk that absorbs the stellar radiation and re-radiates it in the IR, without taking into account accretion radiation; and model 3 - a central star, a passive disk, and an inner disk hole.

The SEDs have been constructed for the 401 stars in our sample that were modeled with the three model sets. The observational fluxes, from the U band to the $24 \mu \mathrm{m}$ MIPS band taken from the literature, were not dereddened. The input parameters of the models are the fluxes and photometric apertures, a range of $A_{\mathrm{V}}$, and the distance of the star to the Sun. We used the individual value of $A_{\mathrm{V}}$ to each star from Venuti et al. (2014). We used the individual distance values estimated from parallax data obtained from the Gaia mission second release (Gaia Collaboration 2016, 2018). The distance values were calculated using Bayesian methods, following Luri et al. (2018). The individual distance values for our sample of stars are listed in Tables $1-3$. The $A_{\mathrm{v}}$ and the distance uncertainty of each star were used to define the range of $A_{\mathrm{V}}$ and distances to be searched for during the SED modeling. Within the allowed ranges, the SED program can choose the $A_{\mathrm{V}}$ and distance of each system that correspond to the best fit to the observed flux data. For the systems

\footnotetext{
In this work, we used version v1.0 of the code, available to download at https: //doi .org/10.5281/zenodo. 235786.

2 All the modular sets of synthetic spectral energy distributions were downloaded from http://doi.org/10.5281/zenodo. 166732 , using version v1.1 of the models.
} 
Table 1. Observational parameters of our sample of transition disk candidates.

\begin{tabular}{|c|c|c|c|c|c|c|c|c|c|c|c|c|c|}
\hline Mon ID ${ }^{(a)}$ & TwoMass ID $^{(b)}$ & $\mathrm{SpT}^{(c)}$ & $\begin{array}{l}\mathrm{EW}_{\mathrm{H} \alpha}{ }^{(d)} \\
(\AA)\end{array}$ & $\begin{array}{l}\mathrm{W} 10 \%_{\mathrm{H} \alpha}(d) \\
\left(\mathrm{km} \mathrm{s}^{-1}\right)\end{array}$ & $\begin{array}{l}\mathrm{EW}_{\mathrm{H} \alpha}{ }^{(e)} \\
(\AA)\end{array}$ & $\operatorname{Class}^{(f)}$ & $\alpha_{\mathrm{IRAC}}{ }^{(g)}$ & $\begin{array}{l}R_{\star}{ }^{(h)} \\
\left(R_{\odot}\right) \\
\end{array}$ & $\begin{array}{l}T_{\star}{ }^{(h)} \\
(\mathrm{K}) \\
\end{array}$ & $\begin{array}{l}\mathrm{UV}_{\text {Exc. }}{ }^{(h)} \\
\text { (mag) }\end{array}$ & $\begin{array}{l}\dot{M}_{\mathrm{UV}_{\mathrm{Exc}}}{ }^{(h)} \\
\left(M_{\odot} \mathrm{yr}^{-1}\right)\end{array}$ & $\begin{array}{l}\dot{M}_{\mathrm{H} \alpha}{ }^{(d)} \\
\left(M_{\odot} \mathrm{yr}^{-1}\right)\end{array}$ & $\begin{array}{l}\text { Dist }{ }^{(i)} \\
(\mathrm{pc})\end{array}$ \\
\hline Mon-000040 & $06410892+0956476$ & M4.5 & & & & $\mathrm{c}$ & -2.29 & 0.85 & 3175. & -0.340 & $4.3 \mathrm{E}-10$ & & 664.7 \\
\hline Mon-000120 & $06411911+0926294$ & M3 & & & 155.9 & $\mathrm{c}$ & -2.12 & 2.09 & 3360. & -1.930 & $1.7 \mathrm{E}-07$ & & 749.3 \\
\hline Mon-000122 & $06414711+0938047$ & K6 & & & 1.4 & w & -2.84 & 1.57 & 4200. & -0.190 & & & 714.3 \\
\hline Mon-000177 & $06410620+0936229$ & G5 & 11.1 & 412 & 10.0 & $\mathrm{c}$ & -2.03 & 2.03 & 5660. & 0.067 & & $9.3 \mathrm{E}-09$ & 703.0 \\
\hline Mon-000273 & $06411837+0939411$ & M1 & & & 123.5 & $\mathrm{c}$ & -2.28 & 1.60 & 3680. & -1.050 & $2.1 \mathrm{E}-08$ & & 560.4 \\
\hline Mon-000280 & $06404100+0927543$ & K4 & 12.8 & 486 & 13.2 & $\mathrm{c}$ & -1.97 & 1.07 & 4580. & -0.090 & $8.9 \mathrm{E}-10$ & $5.9 \mathrm{E}-10$ & 741.1 \\
\hline Mon-000296 & $06405059+0954573$ & K2 & 10.6 & 375 & 11.2 & $\mathrm{c}$ & -2.14 & 1.71 & 4950. & -0.084 & & $1.7 \mathrm{E}-09$ & 741.7 \\
\hline Mon-000314 & $06404459+0932261$ & M3 & 53.2 & 287 & 60.0 & $\mathrm{c}$ & -2.61 & 31.35 & 3360. & -1.230 & $9.5 \mathrm{E}-09$ & $1.1 \mathrm{E}-08$ & 751.7 \\
\hline Mon-000328 & $06412700+0930131$ & M1 & 31.9 & 231 & 25.8 & $\mathrm{c}$ & -1.88 & 1.34 & 3680. & -0.340 & $2.4 \mathrm{E}-09$ & $3.9 \mathrm{E}-09$ & 755.4 \\
\hline Mon-000342 & $06405573+0946456$ & M4 & & & 21.1 & $\mathrm{c}$ & -1.79 & 1.57 & 3230. & -1.270 & $1.7 \mathrm{E}-08$ & & 946.9 \\
\hline Mon-000433 & $06410111+0934522$ & M1 & & & 7.0 & $\mathrm{c}$ & -2.24 & 30.99 & 3680. & -0.100 & $1.4 \mathrm{E}-09$ & & 766.3 \\
\hline Mon-000452 & $06410160+0937287$ & M2.5 & & & 154.8 & $\mathrm{c}$ & -1.71 & 0.92 & 3430. & -2.340 & $1.9 \mathrm{E}-08$ & & 829.2 \\
\hline Mon-000502 & $06405777+0930502$ & K7 & & & 7.3 & $\mathrm{w}$ & -2.37 & 2.03 & 4000. & -0.033 & & & 663.7 \\
\hline Mon-000637 & $06404921+0957387$ & M1 & & & 50.8 & $\mathrm{c}$ & -2.67 & 1.64 & 3680. & -0.420 & $3.2 \mathrm{E}-09$ & & 720.1 \\
\hline Mon-000676 & $06414780+0934096$ & K5 & & & & $\mathrm{w}$ & -1.53 & 2.32 & 4395. & 0.517 & & & 728.8 \\
\hline Mon-000771 & $06411827+0933535$ & K4 & & & 28.9 & $\mathrm{c}$ & -2.39 & 1.83 & 4580. & -0.270 & & & 762.9 \\
\hline Mon-000824 & $06410183+0938411$ & K4 & -0.5 & 493 & 1.5 & $\mathrm{c}$ & -2.41 & 2.23 & 4580. & -0.068 & & $3.3 \mathrm{E}-09$ & 606.9 \\
\hline Mon-000860 & $06415492+0942527$ & M2.5 & & & 261.0 & $\mathrm{c}$ & -3.23 & 1.18 & 3430. & -0.900 & $5.6 \mathrm{E}-09$ & & 708.7 \\
\hline Mon-000879 & $06410338+0940448$ & M1 & & & 16.5 & $\mathrm{c}$ & -2.69 & 1.99 & 3680. & -0.130 & $1.2 \mathrm{E}-08$ & & 1027.0 \\
\hline Mon-000937 & $06405255+0952059$ & K7 & 8.5 & 371 & 6.9 & $\mathrm{c}$ & -2.40 & 2.10 & 4000. & -0.010 & $1.0 \mathrm{E}-08$ & 7.2E -10 & 890.7 \\
\hline Mon-000961 & $06411247+0908509$ & M3.5 & & & & $\mathrm{w}$ & -1.20 & 0.90 & 3300. & 0.010 & $7.9 \mathrm{E}-11$ & & 706.1 \\
\hline Mon-000965 & $06404600+0917582$ & M2 & 14.4 & 305 & & $\mathrm{c}$ & -2.51 & 1.72 & 3500. & -0.540 & $1.0 \mathrm{E}-08$ & $1.8 \mathrm{E}-09$ & 886.9 \\
\hline Mon-000997 & $06403077+0948076$ & M1 & & & 72.8 & $\mathrm{c}$ & -4.22 & 0.85 & 3680. & -0.120 & $2.8 \mathrm{E}-10$ & & \\
\hline Mon-001033 & $06404102+0947577$ & K7 & 6.3 & 434 & 7.5 & $\mathrm{c}$ & -1.97 & 1.67 & 4000 & -0.700 & $2.0 \mathrm{E}-08$ & 4.7E-09 & 737.2 \\
\hline Mon-001094 & $06403164+0948233$ & K5 & & & 54.6 & $\mathrm{c}$ & -1.96 & 1.49 & 4395. & -0.670 & $1.6 \mathrm{E}-08$ & & 750.2 \\
\hline Mon-001229 & $06403697+0939097$ & M1 & & & 5.0 & $\mathrm{w}$ & -1.92 & 1.46 & 3680. & -0.248 & & & 753.1 \\
\hline Mon-001287 & $06401110+0921270$ & M3 & 126.0 & 315 & 163.1 & $\mathrm{c}$ & -1.62 & 1.08 & 3360. & -1.930 & $1.9 \mathrm{E}-08$ & & 795.2 \\
\hline Mon-001308 & $06395924+0927245$ & M0 & & & & $\mathrm{c}$ & -1.87 & 1.59 & 3920. & -0.800 & $1.9 \mathrm{E}-08$ & & 744.9 \\
\hline
\end{tabular}

Notes. This table is ordered according to the Mon ID. ${ }^{(a)}$ CSIMon is an internal identification of the CSI 2264 campaign. Here, CSI was omitted for brevity. ${ }^{(b)}$ 2Mass identification. ${ }^{(c)}$ Spectral type obtained by Venuti et al. (2014). ${ }^{(d)} \mathrm{H} \alpha$ parameters obtained using FLAMES spectra by Sousa et al. (2016). They used the convention that positive $\mathrm{H} \alpha$ equivalent width indicates $\mathrm{H} \alpha$ in emission, and negative values correspond to $\mathrm{H} \alpha$ in absorption. ${ }^{(e)} \mathrm{H} \alpha$ equivalent width obtained by Dahm \& Simon (2005). ${ }^{(f)}$ Classification as CTTS (c) and WTTS (w) by Sousa et al. (2016) and Venuti et al. ${ }^{(2014) .}{ }^{\left({ }^{(g)}\right.} \alpha_{\text {IRAC }}$ is the slope of the SED between $3.6 \mu \mathrm{m}$ and $8 \mu \mathrm{m}$ obtained by Teixeira et al. (2012). ${ }^{(h)}$ Parameters obtained using CFHT data by Venuti et al. (2014). ${ }^{(i)}$ Distance from the Sun which was obtained from the Gaia parallax data, following (Luri et al. 2018).

that did not have $A_{\mathrm{v}}$ and/or distance (Gaia parallax) available, we chose the values that represented a best fit to the data. We used the same extinction law described in Robitaille et al. (2007). In the model calculations, the inner disk radius $\left(R_{\text {in }}\right)$ is set to the dust sublimation radius $\left(R_{\text {sub }}\right)$ in disks without an inner hole (model 2) or varied from 1 to $1000 R_{\text {sub }}$ (Robitaille 2017) to reach a best fit, when an inner disk hole is present (model 3).

Among the 401 TTS in our sample, only 152 have detections available at $22 \mu \mathrm{m}$ (WISE) or $24 \mu \mathrm{m}$ (MIPS), which are important for constraining the outer disk. Without these data, the SED fitting is highly degenerate in the outer disk region. The MIPS observations targeted preferentially stars that presented IR excess; we therefore expect that stars which were not detected in these bands to have little or no excess at these wavelengths (Teixeira et al. 2012).

The SED models were calculated for each source and the different output models were compared with the observational data through a $\chi^{2}$ test. The best model was the one presenting the minimum $\chi^{2}$ value. For the 152 stars that had $22 \mu \mathrm{m}$ or $24 \mu \mathrm{m}$ detections, we calculated models 2 and 3 , choosing the best model that fit the data and showed output stellar parameters (temperature and radii) from the fitting procedure close to the values available in the literature. A total of 249 stars did not have $24 \mu \mathrm{m}$ and/or $22 \mu \mathrm{m}$ measurements or have only an upper limit at these wavelengths. Among those systems, we fit models 2 and 3 to the 85 stars that presented excess in the inner disk (based in the $\alpha_{\text {IRAC }}$ index) or signs of accretion (based in the $\mathrm{H} \alpha$ line analyze Sousa et al. 2016 or UV excess Venuti et al. 2014). The 164 stars without IR excess and presenting no accretion signature were fit with model 1, composed only of a stellar photosphere. The sample of diskless stars was used to compare its characteristics with the sample of transition disk candidates. Among the 401 TTS in our sample, the SEDs of 7 stars could not be fit by any reasonable physical model, sometimes due to a large discrepancy between the temperature or radius of the best model compared to the literature values or the best model did not produce a good fit to the observational data.

In Fig. 1 we show a comparison of the $A_{\mathrm{V}}$, distance, stellar radii, and stellar temperatures found by Hyperion SED fitting model with the values by Venuti et al. (2014) and the distances calculated using Gaia parallax data following Luri et al. (2018). As $A_{\mathrm{V}}$ and the distances are input parameters of the model, the values returned by the model agree with the literature value. The stellar temperature and radius are output parameters and the bestfit values are not always accurate, but for most sources, the deviations from the literature values are small.

The SEDs have been extensively used in the literature to identify transition disks (e.g., Calvet et al. 2005; Merín et al. 2010; Owen 2016; Grant et al. 2018). We used the SED to measure the near-IR and mid-IR flux excess (Strom et al. 1989) and the model including an inner hole to confirm or refute the presence of a hole in the inner disk (e.g., Merín et al. 2010). In this work, we selected as transition disk candidates stars with innerdisk holes according to the SED fitting (systems with $R_{\text {in }}>R_{\text {sub }}$ ). We also only selected systems that have $24 \mu \mathrm{m}$ flux detected 
Table 2. Observational parameters of our sample of full disk systems.

\begin{tabular}{|c|c|c|c|c|c|c|c|c|c|c|c|c|c|}
\hline Mon ID $^{(a)}$ & TwoMass ID $^{(b)}$ & $\mathrm{SpT}^{(c)}$ & $\begin{array}{l}\mathrm{EW}_{\mathrm{H} \alpha}{ }^{(d)} \\
(\AA)\end{array}$ & $\begin{array}{l}\mathrm{W} 10 \%_{\mathrm{H} \alpha}(d) \\
\left(\mathrm{km} \mathrm{s}^{-1}\right)\end{array}$ & $\begin{array}{l}\mathrm{EW}_{\mathrm{H} \alpha}{ }^{(e)} \\
(\AA)\end{array}$ & Class $(f)$ & $\alpha_{\mathrm{IRAC}}{ }^{(g)}$ & $\begin{array}{l}R_{\star}{ }^{(h)} \\
\left(R_{\odot}\right)\end{array}$ & $\begin{array}{l}T_{\star}{ }^{(h)} \\
(\mathrm{K}) \\
\end{array}$ & $\begin{array}{l}\mathrm{UV}_{\text {Exc. }}{ }^{(h)} \\
(\mathrm{mag})\end{array}$ & $\begin{array}{l}\dot{M}_{\mathrm{UV}_{\text {Exe. }}}{ }^{(h)} \\
\left(M_{\odot} \mathrm{yr}^{-1}\right)\end{array}$ & $\begin{array}{l}\dot{M}_{\mathrm{H} \alpha}{ }^{(d)} \\
\left(M_{\odot} \mathrm{yr}^{-1}\right)\end{array}$ & $\begin{array}{l}\text { Dist }^{(i)} \\
(\mathrm{pc})\end{array}$ \\
\hline Mon-000007 & $06415304+0958028$ & K7 & & & & $\mathrm{c}$ & -1.47 & & 4000 & -0.650 & $6.0 \mathrm{E}-08$ & & \\
\hline Mon-000017 & $06413199+1000244$ & K5 & & & 13.1 & $\mathrm{c}$ & -2.69 & 1.45 & 4395. & -0.270 & $5.8 \mathrm{E}-09$ & & 694.0 \\
\hline Mon-000028 & $06410511+0958461$ & M3.5 & & & 294.5 & $\mathrm{c}$ & -1.64 & 0.57 & 3300. & -1.400 & $7.4 \mathrm{E}-10$ & & 705.8 \\
\hline Mon-000056 & $06415315+0950474$ & K5 & & & 1.8 & $\mathrm{c}$ & -1.36 & 1.52 & 4395. & 0.010 & $5.8 \mathrm{E}-09$ & & 756.8 \\
\hline Mon-000059 & $06410334+1000478$ & M4 & & & & $\mathrm{c}$ & -1.44 & 0.76 & 3230 . & -1.110 & $1.2 \mathrm{E}-09$ & & 788.8 \\
\hline Mon-000063 & $06411193+0959412$ & M2.5 & & & 19.4 & $\mathrm{c}$ & -1.37 & 1.19 & 3430. & -1.030 & $6.2 \mathrm{E}-09$ & & 748.9 \\
\hline Mon-000064 & $06411070+0957424$ & M1.5 & & & 3.5 & $\mathrm{w}$ & -1.15 & 1.62 & 3590. & -0.320 & 4.7E-09 & & 736.4 \\
\hline Mon-000080 & $06411795+1004021$ & M3 & & & & $\mathrm{w}$ & -1.12 & 1.17 & 3360. & -0.460 & $1.9 \mathrm{E}-09$ & & 723.2 \\
\hline Mon-000081 & $06405978+1002126$ & M2 & & & 155.0 & $\mathrm{c}$ & -1.01 & 1.00 & 3500. & -1.340 & $5.4 \mathrm{E}-09$ & & 667.7 \\
\hline Mon-000090 & $06410896+0933460$ & M3 & & & 51.0 & $\mathrm{c}$ & -0.88 & 2.26 & 3360. & -0.550 & $1.1 \mathrm{E}-08$ & & 786.6 \\
\hline Mon-000099 & $06411610+0926435$ & M4.5 & & & & $\mathrm{w}$ & -1.44 & 1.16 & 3175 . & 0.360 & $1.1 \mathrm{E}-10$ & & 743.3 \\
\hline Mon-000103 & $06405954+0935109$ & K6 & 15.6 & 401 & 6.4 & $\mathrm{c}$ & -1.14 & 3.84 & 4200. & 0.591 & & $9.6 \mathrm{E}-09$ & 658.2 \\
\hline Mon-000106 & $06405272+0928437$ & M0.5 & & & & $\mathrm{c}$ & -1.40 & 0.74 & 3800. & -1.260 & $1.3 \mathrm{E}-09$ & & 764.8 \\
\hline Mon-000113 & $06410028+0928341$ & K6 & & & 314.6 & $\mathrm{c}$ & -1.25 & 0.71 & 4200. & -2.380 & $6.3 \mathrm{E}-09$ & & 672.4 \\
\hline Mon-000117 & $06405413+0948434$ & M2.5 & & & 353.0 & $\mathrm{c}$ & -2.34 & 1.21 & 3430. & -2.090 & $3.4 \mathrm{E}-08$ & & 752.9 \\
\hline Mon-000119 & $06412100+0933361$ & K6 & 10.5 & 466 & 10.6 & $\mathrm{c}$ & -1.44 & 2.04 & 4200. & -0.147 & & $3.3 \mathrm{E}-09$ & \\
\hline Mon-000126 & $06405783+0941201$ & M0 & & & 26.4 & $\mathrm{c}$ & -1.09 & 2.02 & 3920. & -0.540 & $2.1 \mathrm{E}-08$ & & 573.7 \\
\hline Mon-000136 & $06411599+0926094$ & M0 & & & 174.1 & $\mathrm{c}$ & -0.53 & 1.99 & 3920. & -1.150 & $1.6 \mathrm{E}-08$ & & 792.4 \\
\hline Mon-000137 & $06404712+0942077$ & M2 & & & 11.4 & $\mathrm{c}$ & -1.54 & 1.36 & 3500. & -0.360 & $2.9 \mathrm{E}-09$ & & 557.9 \\
\hline Mon-000145 & $06412346+0945586$ & K7 & & & 4.0 & $\mathrm{w}$ & -2.46 & 1.39 & 4000 & 0.050 & & & 820.3 \\
\hline Mon-000153 & $06405990+0947044$ & M3 & & & 39.9 & $\mathrm{c}$ & -1.71 & 1.35 & 3360. & -1.220 & $9.1 \mathrm{E}-09$ & & 791.7 \\
\hline Mon-000165 & $06410481+0944333$ & $\mathrm{~K} 4.5$ & & & 92.9 & $\mathrm{c}$ & -0.62 & 0.76 & 4490. & -1.580 & $1.5 \mathrm{E}-09$ & & 743.8 \\
\hline Mon-000168 & $06414287+0925084$ & K5:M0 & 44.9 & 390 & 86.0 & $\mathrm{c}$ & -1.38 & 1.20 & 4157. & -1.450 & $1.1 \mathrm{E}-08$ & $3.8 \mathrm{E}-09$ & 776.0 \\
\hline
\end{tabular}

Notes. Only a portion of this table is shown here. A full version is available at the CDS. This table is ordered according to the Mon ID. ${ }^{(a)}$ CSIMon is an internal identification of the CSI 2264 campaign. Here, CSI was omitted for brevity. ${ }^{(b)} 2$ Mass identification. ${ }^{(c)}$ Spectral type obtained by Venuti et al. (2014). ${ }^{\left({ }^{d}\right)} \mathrm{H} \alpha$ parameters obtained using FLAMES spectra by Sousa et al. (2016). They used the convention that positive H $\alpha$ equivalent width indicates $\mathrm{H} \alpha$ in emission, and negative values correspond to $\mathrm{H} \alpha$ in absorption. ${ }^{(e)} \mathrm{H} \alpha$ equivalent width obtained by Dahm \& Simon (2005). ${ }^{(f)}$ Classification as CTTS (c) and WTTS (w) by Sousa et al. (2016) and Venuti et al. (2014). ${ }^{(g)} \alpha_{\text {IRAC }}$ is the slope of the SED between $3.6 \mu \mathrm{m}$ and $8 \mu \mathrm{m}$ obtained by Teixeira et al. (2012). ${ }^{(h)}$ Parameters obtained using CFHT data by Venuti et al. (2014). ${ }^{(i)}$ Distance from the Sun which was obtained from the Gaia parallax data, following (Luri et al. 2018). The systems Mon-000168, Mon-000358, Mon-000361, Mon-000687, Mon-000753 could be classified as transition disk using transition disk select criteria from Grant et al. (2018), see text.

above the photospheric level larger than the $10 \mu \mathrm{m}$ flux. We found 28 stars that fulfill all these conditions in our sample. Among the remaining systems, 209 stars were modeled with a full disk and 164 as diskless. In Fig. 2 we show some examples of SEDs of diskless stars, transition disk candidates, and full disk systems. The black solid line is the best fit (based on $\chi^{2}$ ) that represents the total flux of the system. The dashed red and blue lines are the components of emission from dust and the central star, respectively. The dust and stellar emission components were computed using the Hyperion $\operatorname{code}^{3}$ (Robitaille 2011), after determining the best the SED model for each system (Robitaille 2017). In Sect. 5.3 we analyze the disk and the inner-disk hole determined values. From here on, the results will be discussed in terms of three groups: diskless stars, transition disk candidates, and full disk systems. In Tables 1-3 we show the observational parameters of the transition disk candidates, full disk systems, and diskless stars, respectively.

\section{Results}

\subsection{Star formation sites in NGC 2264}

Star formation is still ongoing in some regions of NGC 2264 (Lamm et al. 2004; Sung et al. 2009; Teixeira et al. 2012). These regions have the most embedded members $\left(A_{\mathrm{v}}>3.0\right)$ in the cluster, as seen in Teixeira et al. (2012). Since the youngest stars

\footnotetext{
3 In this work, we used version 0.9 .8 of the Hyperion code available to download from http://www. hyperion-rt.org and model sets from https://doi .org/10.5281/zenodo. 572233 .
}

are clustered, we wondered if the transition disks were clustered too. We checked if transition disk candidates were randomly located in NGC 2264 or preferentially clustered in some spatial regions.

We show in Fig. 3 the spatial distribution of our three groups of stars. The boxes define the regions of the most active star formation of the cluster taken from Lamm et al. (2004). We calculated the fraction of stars of a given group that falls in the boxes, that is the number of systems of a given group in the boxes divided by the total number of systems in the boxes. To compare with that, we also calculated the fraction of each group that falls out of the boxes, that is the number of systems of a given group out of the boxes divided by the total number of systems out of the boxes. We found that in the boxes, $\sim 59 \%$ of systems are full disks, $\sim 4 \%$ are transition disk candidates, and $\sim 37 \%$ correspond to diskless stars. The fractions of systems that fall out of the boxes are $\sim 49 \%$ full disks, $\sim 8 \%$ transition disk candidates, and $\sim 43 \%$ diskless stars. The population in the active star formation regions are predominantly composed of full disk systems and the fraction of transition disk candidates and diskless stars in these regions is considerably smaller than outside, which may point to an evolutionary scenario for the cluster, as discussed by Venuti et al. (2014).

\subsection{Disk diagnostics}

Transition disks have a dust hole and gap in the inner disk region, characterized by a lower quantity of dust compared to the outer disk. Therefore, we expect that transition disks will show less excess in near-IR than full disks, while the excess at 
Table 3. Observational parameters of our sample of diskless stars.

\begin{tabular}{|c|c|c|c|c|c|c|c|c|c|c|c|c|}
\hline $\operatorname{Mon~ID~}^{(a)}$ & TwoMass ID $^{(b)}$ & $\mathrm{SpT}^{(c)}$ & $\begin{array}{l}\mathrm{EW}_{\mathrm{H} \alpha}{ }^{(d)} \\
(\AA)\end{array}$ & $\begin{array}{l}\mathrm{W} 10 \%_{\mathrm{H} \alpha}(d) \\
\left(\mathrm{km} \mathrm{s}^{-1}\right)\end{array}$ & $\begin{array}{l}\mathrm{EW}_{\mathrm{H} \alpha}{ }^{(e)} \\
(\AA)\end{array}$ & $\operatorname{Class}^{(f)}$ & $\alpha_{\mathrm{IRAC}}{ }^{(g)}$ & $\begin{array}{l}R_{\star}{ }^{(h)} \\
\left(R_{\odot}\right) \\
\end{array}$ & $\begin{array}{l}T_{\star}{ }^{(h)} \\
(\mathrm{K}) \\
\end{array}$ & $\begin{array}{l}\mathrm{UV}_{\text {Exc. }}(h) \\
(\mathrm{mag})\end{array}$ & $\begin{array}{l}\dot{M}_{\mathrm{H} \alpha}{ }^{(d)} \\
\left(M_{\odot} \mathrm{yr}^{-1}\right)\end{array}$ & $\begin{array}{l}\text { Dist }{ }^{(i)} \\
(\mathrm{pc})\end{array}$ \\
\hline Mon-000018 & $06411322+0955086$ & $\mathrm{~K} 3: \mathrm{K} 4$ & & & 3.0 & w & -2.73 & 2.03 & 4630. & 0.216 & & 812.9 \\
\hline Mon-000023 & $06411242+0955001$ & M3 & & & & $\mathrm{w}$ & -2.74 & 0.98 & 3360. & -0.011 & & 699.7 \\
\hline Mon-000029 & $06410328+0957549$ & K7 & & & 1.5 & $\mathrm{w}$ & -2.85 & 1.68 & 4000. & -0.040 & & 706.5 \\
\hline Mon-000033 & $06410726+0958311$ & K5 & & & 1.4 & w & -2.78 & 1.87 & 4395. & -0.046 & & 723.1 \\
\hline Mon-000050 & $06410153+1000365$ & K4 & & & 1.1 & w & -2.88 & 1.47 & 4580. & -0.081 & & 746.5 \\
\hline Mon-000055 & $06413491+1001472$ & M2 & & & & $\mathrm{w}$ & -2.80 & 1.53 & 3500. & -0.111 & & 711.3 \\
\hline Mon-000057 & $06410393+0958094$ & M3 & & & 4.1 & $\mathrm{w}$ & -2.74 & 2.16 & 3360. & 0.172 & & 736.6 \\
\hline Mon-000060 & $06411532+0954509$ & M3 & & & 4.0 & w & -2.92 & 1.25 & 3360. & 0.016 & & 998.3 \\
\hline Mon-000066 & $06410357+1000353$ & M1 & & & 6.9 & $\mathrm{w}$ & -2.66 & 1.53 & 3680. & 0.027 & & 760.0 \\
\hline Mon-000075 & $06411159+1002235$ & K4 & & & & w & -2.84 & 2.18 & 4580. & 0.079 & & 762.9 \\
\hline Mon-000086 & $06404645+0959463$ & M0 & & & & $\mathrm{w}$ & -3.04 & 1.45 & 3920. & 0.221 & & 717.2 \\
\hline Mon-000104 & $06410417+0952020$ & K6 & & & 1.3 & w & -2.79 & 1.87 & 4200. & 0.010 & & 763.1 \\
\hline Mon-000108 & $06411484+0932358$ & M3 & & & 2.2 & $\mathrm{w}$ & -2.85 & 3.00 & 3360. & 0.235 & & 883.5 \\
\hline Mon-000121 & $06414901+0941061$ & M0 & & & 1.7 & w & -2.72 & 1.95 & 3920. & 0.200 & & 923.2 \\
\hline Mon-000135 & $06405999+0928500$ & K7 & & & 1.7 & w & -2.59 & 1.64 & 4000 & 0.009 & & 752.9 \\
\hline Mon-000139 & $06405367+0958000$ & M1 & & & 3.2 & $\mathrm{w}$ & -3.16 & 1.36 & 3680. & -0.145 & & 709.2 \\
\hline Mon-000142 & $06404480+0949478$ & M2 & & & 5.5 & $\mathrm{w}$ & -3.01 & 1.13 & 3500. & 0.100 & & 726.1 \\
\hline Mon-000143 & $06410454+0926092$ & M3 & & & 3.8 & $\mathrm{w}$ & -2.80 & 1.03 & 3360. & -0.000 & & 772.1 \\
\hline Mon-000149 & $06411330+0951544$ & M1 & & & 2.0 & w & -2.69 & 1.25 & 3680. & -0.081 & & 733.5 \\
\hline Mon-000151 & $06411829+0928330$ & M0 & & & 6.2 & $\mathrm{w}$ & -2.66 & 0.94 & 3920. & 0.030 & & 759.8 \\
\hline Mon-000158 & $06404484+0946384$ & K0.5 & & & & $\mathrm{w}$ & -3.80 & 1.05 & 5170. & -0.626 & & 660.7 \\
\hline Mon-000159 & $06405146+0937144$ & M1 & & & 3.4 & w & -4.65 & 1.38 & 3680. & 0.055 & & 701.9 \\
\hline
\end{tabular}

Notes. Only a portion of this table is shown here. A full version is available at the CDS. This table is ordered according to the Mon ID. ${ }^{(a)}$ CSIMon is an internal identification of the CSI 2264 campaign. Here, CSI was omitted for brevity. ${ }^{(b)}$ 2Mass identification. ${ }^{(c)}$ Spectral type obtained by Venuti et al. (2014). ${ }^{(d)} \mathrm{H} \alpha$ parameters obtained using FLAMES spectra by Sousa et al. (2016). They used the convention that positive H $\alpha$ equivalent width indicates $\mathrm{H} \alpha$ in emission, and negative values correspond to $\mathrm{H} \alpha$ in absorption. The accretion rate attributed to WTTS, here represented by the diskless sample, corresponds to a contribution from nebular and chromospheric $\mathrm{H} \alpha$ line emission. ${ }^{(e)} \mathrm{H} \alpha$ equivalent width obtained by Dahm $\&$ Simon (2005). ${ }^{(f)}$ Classification as CTTS (c) and WTTS (w) by Sousa et al. (2016) and Venuti et al. (2014). ${ }^{(g)} \alpha_{\text {IRAC }}$ is the slope of the SED between $3.6 \mu \mathrm{m}$ and $8 \mu \mathrm{m}$ obtained by Teixeira et al. (2012). ${ }^{(h)}$ Parameters obtained using CFHT data by Venuti et al. (2014). ${ }^{(i)}$ Distance from the Sun which was obtained from the Gaia parallax data, following (Luri et al. 2018).

longer wavelengths may be compatible with full disk systems. In Fig. 4, we show $K_{s}-[8.0]$ vs. $K_{s}-[24]$ diagrams. The stellar magnitudes were dereddened with individual $A_{\mathrm{v}}$ value from Venuti et al. (2014) and the $A_{\lambda} / A_{\mathrm{v}}$ relation from the SVO Filter ${ }^{4}$, which uses the extinction law by Fitzpatrick (1999), improved by Indebetouw et al. (2005) in the IR. We can see that stars with full disks present excess above the photospheric emission in the inner and outer parts of the disk, while transition disks present emission in the outer disk compatible with a full disk system and lower emission in the near IR than full disks.

As a star moves from a full disk to a diskless system, it moves in different ways in the IR color-color diagrams in Fig. 4. Disk dispersal can occur from outside to inside due to some external factor like photoevaporation by a close high mass star (Scally $\&$ Clarke 2001). These systems will present emission above the photospheric level in the inner disk and little emission in the outer disk. The disk can also be dispersed homogeneously throughout its radius. In this case, the disk will evolve through a diagonal path in Fig. 4, the inner and outer disks presenting the same emission above the photospheric level at each epoch. However, Koepferl et al. (2013) show that this type of disk dispersal is not common. Disk dispersal can also proceed from inside to outside due, for example, to photoevaporation by high-energy radiation of the central star and planet formation. In Fig. 4, these systems should present IR excesses in the outer disk, and weak or no IR excesses in the inner disk.

In Fig. 4, we plot lines from theoretical models that separate stars with disks in different evolutionary stages (Koepferl

\footnotetext{
4 The SVO Filter Profile Service. Rodrigo, C., Solano, E., Bayo, A. http://ivoa.net/documents/Notes/SVOFPS/index.html
}

et al. 2013). Our disk classification is in agreement with the theoretical disk evolution obtained by Koepferl et al. (2013), since our systems classified as full disks are predominantly located in the region with primordial disks (represented by letter $\mathrm{A}$ in the plot) and our transition disk candidates are in the region of inside to outside disk evolution (represented by letter D in the plot). We do not have a significant number of systems in regions $C$ and $\mathrm{E}$ that are expected to contain systems with ultra-settled primordial disks, which correspond to flat disks and homogeneously depleted disks, respectively. Unfortunately, we do not have MIPS data for the sample of diskless stars used in this work, which we expect to show no excess emission at all wavelengths, as seen, for example, in Owen (2016), and should be located in region $B$ in the plot.

In the literature there are several criteria to select transition disk candidates (e.g., Fang et al. 2009, 2013; Muzerolle et al. 2010; Merín et al. 2010; Cieza et al. 2010; Ribas et al. 2013), using IR photometric and spectroscopic data. We can compare our selection criteria with those used in some previous works.

Merín et al. (2010), used photometric fluxes in IRAC and MIPS bands to classify a system as a transition disk. In a [3.6] - [8] versus [8] - [24] diagram they considered that systems with $0>[3.6]-[8]<1.1$ and $3.2<[8]-[24]<5.3$ were transition disks, while systems with $1.1<[3.6]-[8]<1.8$ and $3.2<[8]-[24]<5.3$ presented a small excess emission and were classified as pre-transition disk by Kim et al. (2013). Pretransition disks were defined as systems with a gap between an inner and an outer disk (Espaillat et al. 2008). The selection criteria adopted by Fang et al. (2013) are based in the $K_{s}-$ [5.8] versus [8] - [24] diagram. Systems are classified as transition disks if 


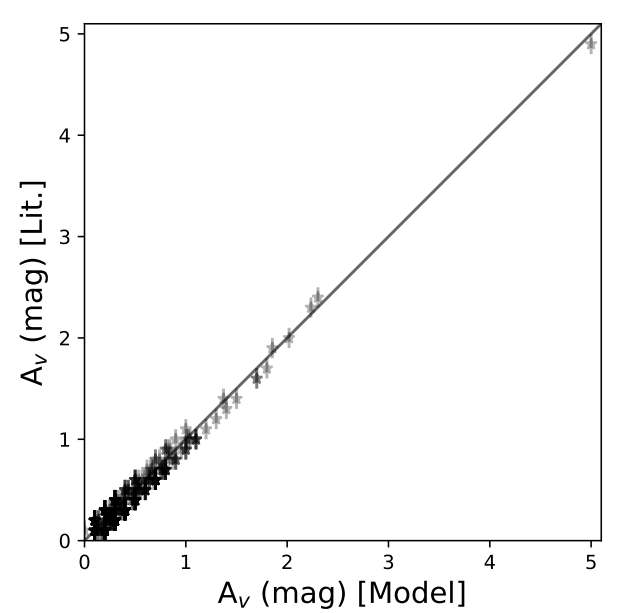

(a)

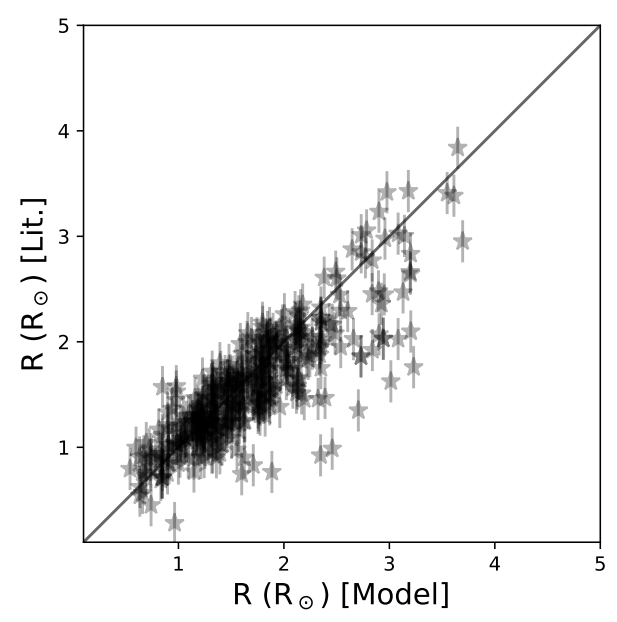

(c)

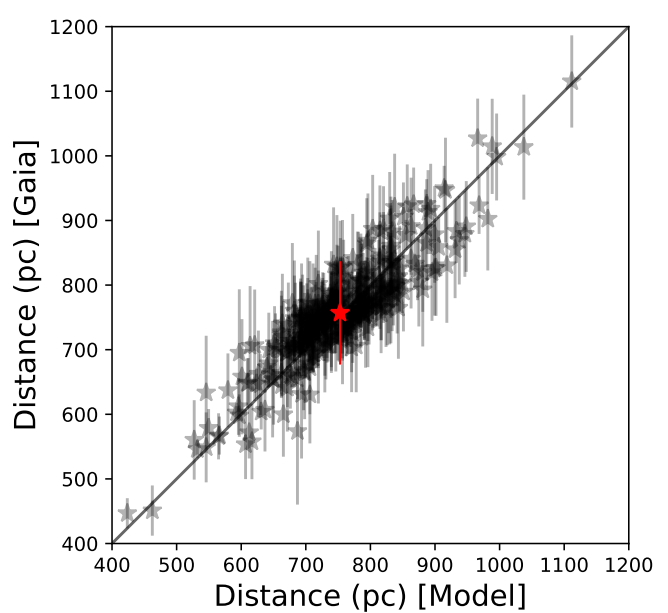

(b)

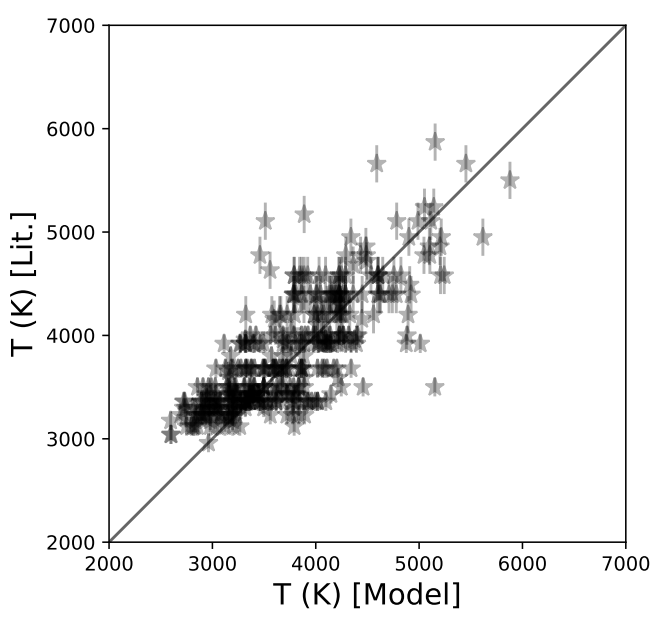

(d)
Fig. 1. Stellar parameters modeled with Hyperion SED fitting code (Robitaille 2017) compared to stellar parameters obtained by Venuti et al. (2014). (a) $A_{\mathrm{V}}$, (b) distance from the Sun. The red point and error bars represent the mean and standard deviation of the distance of the cluster members studied in this work $(d=757 \pm 80 \mathrm{pc}),(c)$ stellar radii, $(d)$ stellar temperatures. $A_{\mathrm{V}}$ and distances are input parameters of the code, while stellar radius and temperature are output parameters of the Hyperion SED Model. The distances were obtained from the Gaia parallax data (Luri et al. 2018). The solid line shows a slope equal to 1 . Based on these plots, we conclude that our modeling is doing at least as well as the prior Venuti et al. (2014) modeling.
[8]-[24] $\geq 2.5$ and if $K_{s}-[5.8] \leq 0.56+([8]-[24]) \times 0.15$. Cieza et al. (2010) proposed a less restrictive transition disk selection criteria, using the [3.6] - [24] versus [3.6] - [4.5] diagram, where a system is classified as transition disk if [3.6] - [24] $>1.5$ and [3.6] $-[4.5]<0.25$. Muzerolle et al. (2010) used as transition disk selection criteria the slopes in $\log \left(\lambda F_{\lambda}\right)$ versus $\log (\lambda)$. They proposed the following limits to the slopes: for weak emission sources, $\alpha_{3.6-5.8}<1.8$ and $-1.5 \leq \alpha_{8-24} \leq 0.0$, and for transition disks, $\alpha_{3.6-5.8}<1.8$ and $\alpha>0.0$.

In Fig. 5 we show the selection criteria discussed above and compare them with our selection criteria. We can see that our transition disk sample is consistent with the various selection criteria. But we also see that 52 systems that we classify as full disks agree with the transition disk or pre-transition disk criteria from Merín et al. (2010), Cieza et al. (2010), Fang et al. (2013), and/or Muzerolle et al. (2010). Only 15 of them would be classified as transition disks by at least three of these criteria, following the selection criteria from Grant et al. (2018). We checked the SEDs of these systems and most of them are not compatible with a transition disk SED. Five systems could be classified as transition disks according to their SEDs, but the photometric data are not good enough to confirm such a classification. We marked these five systems in Table 2 .

The $\alpha_{\text {IRAC }}$ index allows a classification of inner disk evolution, as proposed by Lada et al. (2006). They classified star-disk systems as photospheres (no dust in the inner disk) when $\alpha_{\text {IRAC }}<-2.56$, anemic disk (optically thin disk) if $-2.56<\alpha_{\text {IRAC }}<-1.80$, optically thick disk if $-1.80<\alpha_{\text {IRAC }}<$ -0.5 , flat spectrum for systems with $-0.5<\alpha_{\text {IRAC }}<0.5$, and class I sources if $\alpha_{\text {IRAC }}>0.5$.

Anemic disk systems fall in the transition region between stars with and without dust in the inner disk. This type of disk was also classified as an evolved disk by Hernández et al. (2007). Using only the $\alpha_{\text {IRAC }}$ index that was measured by Teixeira et al. (2012), we would have classified $179(\sim 45 \%)$ systems as diskless stars, that correspond to photospheres, $78(\sim 19 \%)$ as transition disk candidates, which correspond to the anemic disks, and $144(\sim 36 \%)$ as full disk systems, which are the systems classified as flat spectra and thick disks.

Our disk classification based on SED modeling is shown in Fig. 6, where we can see the overall good agreement with the $\alpha_{\text {IRAC }}$ disk classification. All the flat spectra and almost all the thick disk systems are indeed classified as full disks according to our SEDs and most of the photospheres correspond to the diskless SED systems. The transition disk candidates are generally found among the anemic disks, but a significant fraction of anemic disks would not be classified as transition disk candidates based on the SED analysis. Among the 78 systems classified as anemic disks, only 31 have available data at $22 / 24 \mu \mathrm{m}$ and 17 of these were classified as transition disk candidates by the SED fitting. The other 47 anemic disks, identified in Fig. 6 , do not have $22 / 24 \mu \mathrm{m}$ data, and we could not properly model 
A. P. Sousa et al.: A study of accretion and disk diagnostics in the NGC 2264 cluster
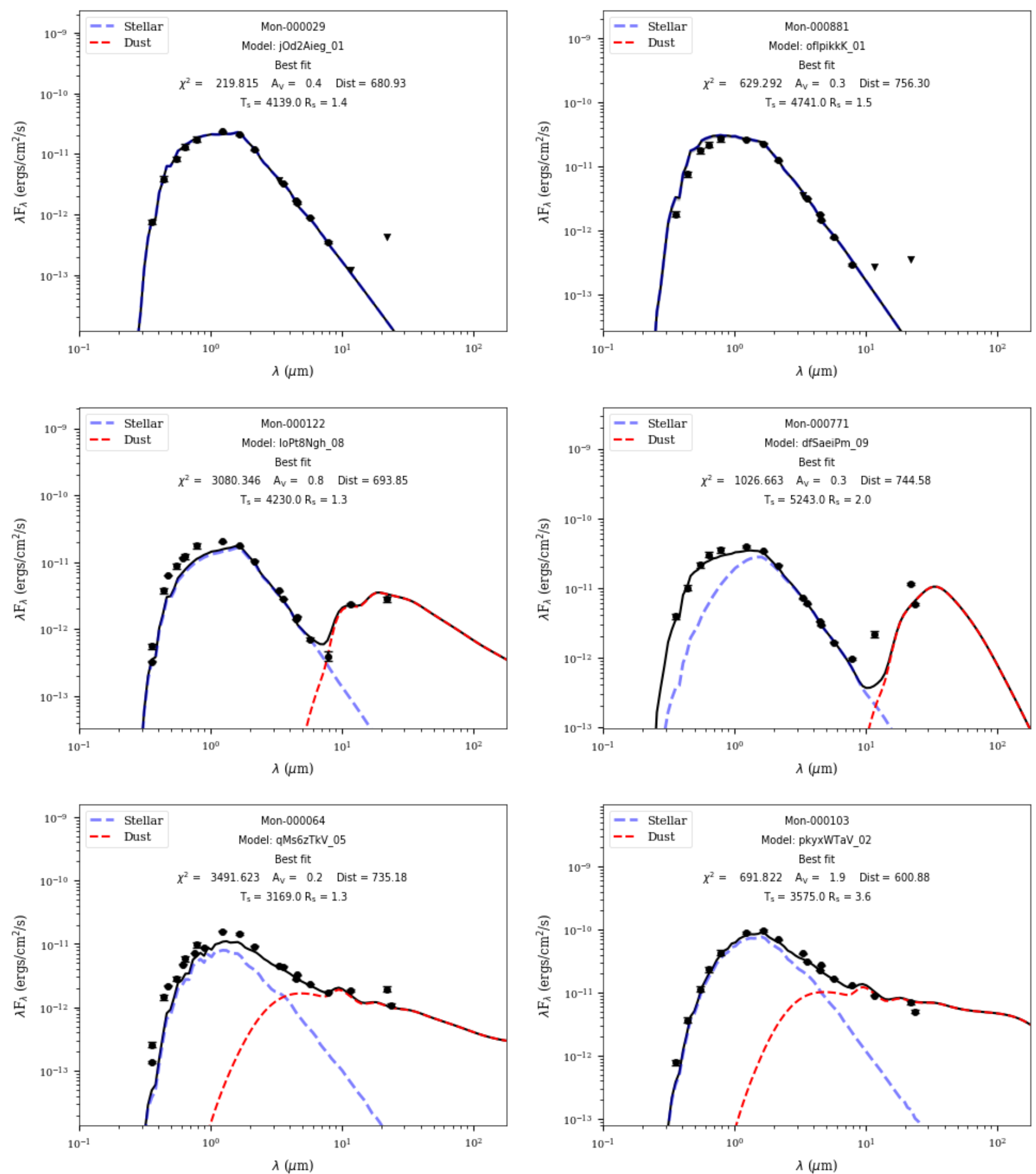

Fig. 2. Examples of synthetic SED fitting, using Hyperion SED code (Robitaille 2017), for our sample of systems classified as diskless stars (top), transition disk candidates (middle) and full disk systems (bottom). The circles show data from the $U$ band ( $0.3 \mu \mathrm{m})$ to the MIPS $24 \mu \mathrm{m}$ band. Triangles, when present, represent the upper limits. The black solid line is the best fit to the SED (Robitaille 2017) and the dashed lines correspond to the stellar (blue) and dust (red) emission components (Robitaille 2011). We found in our sample 7\% transition disk candidates, 52\% systems with full disks, and $41 \%$ diskless stars.

their outer disk emission, as discussed in Sect. 3 and as shown by Robitaille et al. (2007). Therefore the number of transition disk systems in our sample could be larger than what we could determine.

\subsection{Accretion diagnostics}

Accreting young low-mass stars are known as classical T Tauri stars (CTTS) and are characterized by strong and broad emission 


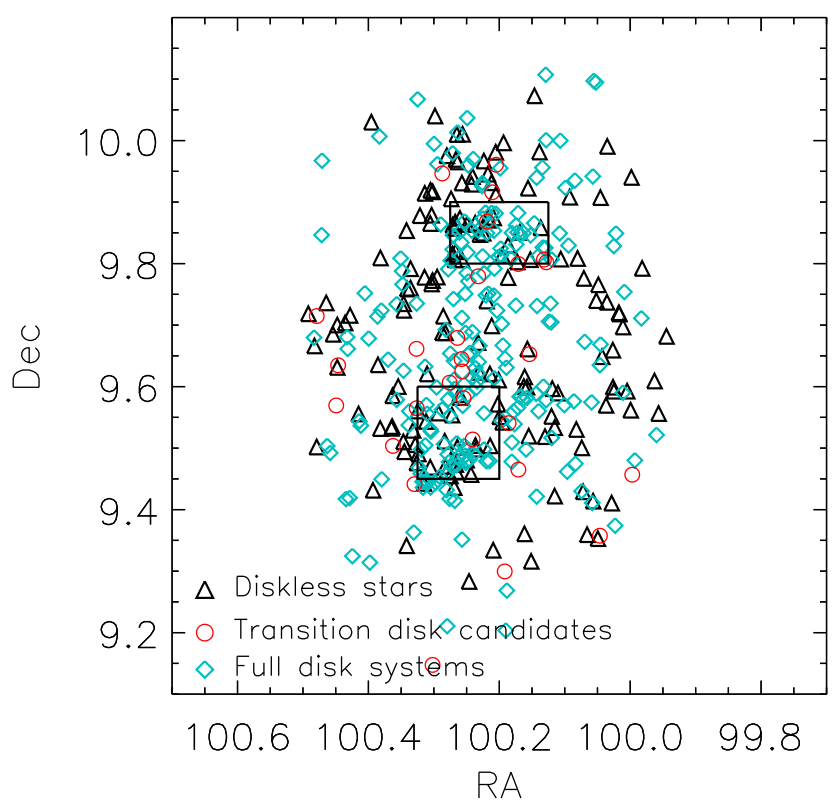

Fig. 3. Spatial distribution of stars belonging to NGC 2264 and analyzed in this work. Our classification of the systems is represented with different symbols and colors. The two boxes delimit the most active star formation regions, as defined by Lamm et al. (2004). The population in these boxes are predominantly composed of full disk systems. Transition disk candidates and diskless stars are preferentially found outside of the active star formation regions.

lines (e.g., $\mathrm{H} \alpha, \mathrm{H} \beta, \mathrm{He} \mathrm{I}$ ) that vary in intensity and morphology as the star-disk system rotates and accretes, and UV excess above the photospheric emission. When the accretion process ceases, the young low-mass stars are called weak line $\mathrm{T}$ Tauri stars (WTTS). Surprisingly, most transition disk systems are found to be accreting (Manara et al. 2014). It is therefore interesting to analyze how the presence of a hole in the inner disk influences accretion to the star in this disk evolutionary phase.

The $\mathrm{H} \alpha$ line is variable, intense and broad in CTTS. This line can be formed in different regions of the star-disk system, such as accretion funnels, winds, and the chromosphere. In typical CTTS, the $\mathrm{H} \alpha$ line emission component is thought to come mostly from the accretion funnel, and it is used as an accretion diagnostic (White \& Basri 2003; Fang et al. 2009; Sousa et al. 2016). The measured equivalent width of $\mathrm{H} \alpha$ depends on the stellar continuum contribution, and is therefore spectral type dependent. For a star to be considered a CTTS, the equivalent width of its $\mathrm{H} \alpha$ line $\left(\mathrm{EW}_{\mathrm{H} \alpha}\right)$ should be larger than $3 \AA$ for spectral types K0-K5, larger than $10 \AA$ for K7-M2.5, larger than $20 \AA$ for M3-M5.5, or larger than $40 \AA$ for M6-M7.5 (White \& Basri 2003). Stars that show $\mathrm{H} \alpha$ width at $10 \%$ of maximum intensity $\left(\mathrm{W} 10 \%_{\mathrm{H} \alpha}\right.$ ) higher than $270 \mathrm{~km} \mathrm{~s}^{-1}$ are also considered CTTS (White \& Basri 2003), since gas at such high velocities cannot be explained by the stellar chromosphere alone.

In Fig. 7 shows the $\mathrm{EW}_{\mathrm{H} \alpha}$ as a function of spectral type and the $\mathrm{W}_{10} \%_{\mathrm{H} \alpha}$ as a function of $\mathrm{EW}_{\mathrm{H} \alpha}$, for full disk systems, transition disk candidates, and diskless stars according to our classification. The $\mathrm{EW}_{\mathrm{H} \alpha}$ were taken from Sousa et al. (2016) and Dahm $\&$ Simon (2005), W10\% $\%_{\mathrm{H} \alpha}$ comes from Sousa et al. (2016), and the spectral types are from Venuti et al. (2014). In Tables 1-3, we show the classification of the stars as CTTS or WTTS for all the systems analyzed in this work. In particular, $\sim 82 \%$ of the transition disk candidates belonging to NGC 2264 still accrete, suggesting that gas is able to flow through the inner disk hole.
In Fig. 7a, we see a considerable number of full disk systems that fall below the $\mathrm{EW}_{\mathrm{H} \alpha}$ threshold of CTTS (blue lines). Among them, 17 stars were observed at $22 / 24 \mu \mathrm{m}$ and have their outer disk emission and their classification as full disk systems well determined. The other 28 full disk systems that fall below the accretion threshold do not have data at $22 / 24 \mu \mathrm{m}$ and their outer disk contribution is not constrained. However, as these systems present emission excess in the inner disk (based on the $\alpha_{\text {IRAC }}$ index obtained by Teixeira et al. 2012), we classified them as full disks. Among these 28 systems, 22 do not present other accretion signatures and are true WTTS, despite having near-IR excess.

In Fig. 8 we show the distribution of $\mathrm{EW}_{\mathrm{H} \alpha}$ and $\mathrm{W}_{10} \%_{\mathrm{H} \alpha}$ of our sample of stars. The $\mathrm{EW}_{\mathrm{H} \alpha}$ mean values are $(3.7 \pm 0.3) \AA$ for diskless stars, $(51 \pm 14) \AA$ for transition disk candidates, and (59 \pm 8$) \AA$ for full disk systems. The transition disk distribution of $\mathrm{EW}_{\mathrm{H} \alpha}$ is more similar to the full disk system distribution than the diskless stars. A two-sided Kolmogorov-Smirnov (K-S) test could not prove that the transition disk and the full disk distributions of $\mathrm{EW}_{\mathrm{H} \alpha}$ are statistically different, with a probability of $98 \%$ to be the same. However, a $\mathrm{K}-\mathrm{S}$ test indicates that the distribution of transition disk candidates and diskless stars are statistically different, with probability of less than $1 \%$ to be the same. The mean values of $\mathrm{W} 10 \%{ }_{\mathrm{H} \alpha}$ are $(172 \pm 10) \AA$ for diskless systems, $(371 \pm 27) \AA$ for transition disk candidates, and $(383 \pm 20) \AA$ for full disk systems. A K-S test shows a probability of $83 \%$ that the distributions of $\mathrm{W} 10 \%_{\mathrm{H} \alpha}$ for transition disks and full disk stars are equal. Comparing the distribution of transition disk candidates with diskless stars, a K-S test shows that they are statistically different with a probability of less then $1 \%$ to be the same.

Using $\mathrm{EW}_{\mathrm{H} \alpha}$, Sousa et al. (2016) calculated the mass accretion rates $\left(\dot{M}_{\mathrm{H} \alpha}\right)$ for a sample of stars belonging to NGC 2264 and observed with the FLAMES spectrograph on VLT. These accretion rates represent a lower limit, because their computation did not take into account absorption due to winds that the $\mathrm{H} \alpha$ line may exhibit, which decreases the total line equivalent width. In Fig. 9, we show $\dot{M}_{\mathrm{H} \alpha}$ from Sousa et al. (2016) as a function of $\mathrm{EW}_{\mathrm{H} \alpha}$ for full disk systems, transition disk candidates and diskless stars. The $\dot{M}_{\mathrm{H} \alpha}$ for transition disk candidates falls between the values computed for full disk systems and diskless stars. The mean $\dot{M}_{\mathrm{H} \alpha}$ value of each group corroborates this analysis: $(9.9 \pm 3.1) \times 10^{-10} M_{\odot} \mathrm{yr}^{-1}$ for diskless stars, $(4.1 \pm 1.2) \times 10^{-9} M_{\odot} \mathrm{yr}^{-1}$ for transition disk candidates, and $(8.2 \pm 0.5) \times 10^{-9} M_{\odot} \mathrm{yr}^{-1}$ for full disk systems. As explained in Manara et al. (2013) and Sousa et al. (2016), the accretion rate attributed to WTTS, here represented by the diskless sample, corresponds to a contribution from nebular and chromospheric $\mathrm{H} \alpha$ line emission.

In CTTS, the stellar magnetic field is expected to be strong enough to truncate the disk at a few stellar radii from the star. The gas accreting from the disk follows the stellar magnetic field and hits the star at high latitudes, creating hot spots at temperatures higher than the stellar photospheric temperature. The UV excess in CTTS comes only from hot spots (e.g., Calvet \& Gullbring 1998; Venuti et al. 2015, 2014), and it is a more direct diagnostic of accretion than $\mathrm{H} \alpha$ line, except for low accretors (Ingleby et al. 2011). UV excesses for the known NGC 2264 members were measured by Venuti et al. (2014) with MegaCam instrument. The UV excess was obtained comparing the observed data with a reference non-accreting threshold defined by the fit of a polynomial function to the locus of non-accreting systems on the $r$ versus $u-r$ color-magnitude diagram. The UV excess was defined as $\mathrm{E}(u)=(u-r)_{\mathrm{obs}}-(u-r)_{\mathrm{ref}}$, where $(u-r)_{\mathrm{obs}}$ is the observed 


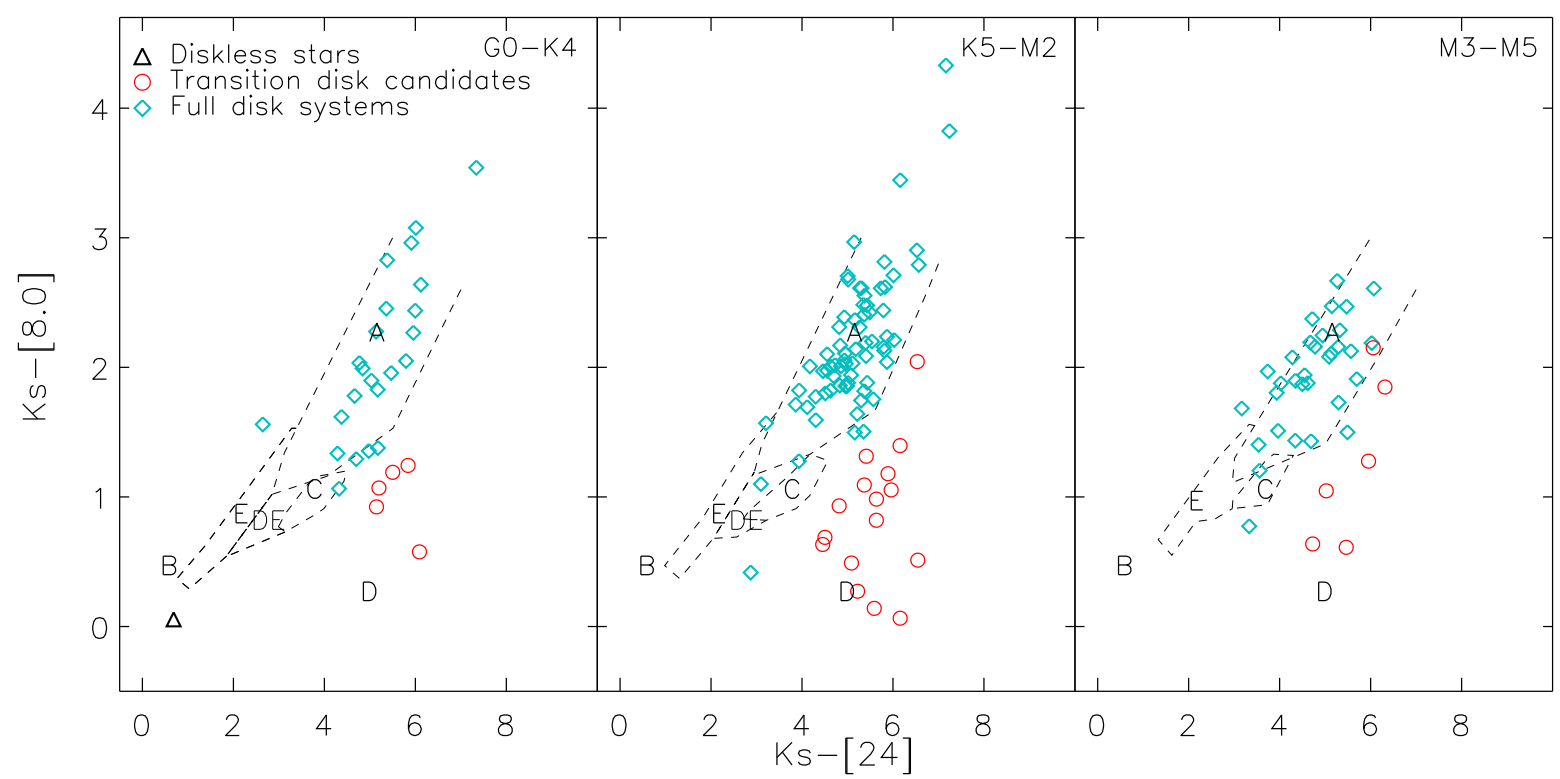

Fig. 4. Near-IR and mid-IR color-color diagram for stars belonging to NGC 2264 broken up by spectral type ranges into three plots. We can see two different populations: stars with full disks present emission excess above the photospheric level in the inner and outer parts of the disk, while transition disk candidates present little dust emission in the inner disk, and dust emission in the outer disk compatible with full disk systems. The dashed lines delimit regions with different disk dispersal mechanisms computed by Koepferl et al. (2013). Region (A) represents thick primordial disks, (B) stellar photospheres, (C) ultra-settled primordial disks (flat disks), (D) inside to outside depleted disks, (E) homogeneously depleted disks and (DE) is a region that may contain systems with homogeneously depleted disks and inside to outside depleted disks. The stars we have selected as full disks and transition disks fall in regions of this diagram that are consistent with that expected from the models.

color of the star and $(u-r)_{\text {ref }}$ is a reference non-accreting color at $r_{\text {obs }}$ magnitude. The more negative the $E(u)$ values, the larger the UV excess.

We show in Fig. 10a the UV excess versus $\mathrm{EW}_{\mathrm{H} \alpha}$. As expected, most stars classified as diskless do not accrete and do not present UV excess. Most full disk systems present UV excess, while the transition disk candidates, in general, present UV excess comparable to full disk systems. The mean UV excess is $0.10 \pm 0.03$ mag for diskless stars, $-0.5 \pm 0.1 \mathrm{mag}$ for transition disk candidates, and $-0.60 \pm 0.06$ mag for full disk systems.

With the UV excess, Venuti et al. (2014) calculated mass accretion rates for a sample of TTS belonging to NGC 2264, shown in Fig. 10b. The mean mass accretion rate values for our sample of stars are $(2 \pm 1) \times 10^{-8} M_{\odot} \mathrm{yr}^{-1}$ for transition disk candidates and $(2.1 \pm 0.4) \times 10^{-8} M_{\odot} \mathrm{yr}^{-1}$ for full disk systems. The mean accretion rates are different from the $\mathrm{H} \alpha$ accretion rates, but also show that the accretion rates of the transition disk candidates are similar to the accretion rates of full disk systems.

\section{Discussion}

\subsection{Photometric period}

The CTTS are contracting and accreting; both physical phenomena should increase the stellar angular momentum. However, CTTS are found to be slow rotators, with typical periods of about 4 to 8 days in young clusters. Some efficient mechanism of angular momentum transfer from the star to the disk or the interstellar medium must then take place. The accretion process is related to the CTTS braking, possibly through disk locking, since at the end of the accretion phase, WTTS start their spin-up toward the Main Sequence (Bouvier et al. 1993; Rebull 2001; Lamm et al. 2005; Cieza et al. 2007; Marilli et al. 2007; Irwin \& Bouvier
2009; Vasconcelos \& Bouvier 2015; Roquette et al. 2017; Venuti et al. 2017; Rebull et al. 2018).

The period distribution of young low-mass stars belonging to NGC 2264 shows that WTTS are substantially more rapid rotators than CTTS. These results were most recently obtained using CoRoT data from the 2008 (Affer et al. 2013) and 2011 (Venuti et al. 2017) campaigns. We analyzed the periodicity of our sample of stars, using the periods obtained by Sousa et al. (2016) for some CTTS and by Venuti et al. (2017) for some CTTS and the WTTS: both papers use observations from the 2011 CoRoT campaign. We complemented our period data with photometric optical periods gathered from the literature in Flaccomio et al. (2006). The period distribution is shown in Fig. 11. Stars with full disks are slower rotators (mean period of $6.0 \pm 0.4$ days) compared to diskless stars (mean period of $4.8 \pm 0.3$ days). This result agrees with the disk locking scenario discussed in the previous paragraph, since most full disk systems are CTTS and all diskless stars are WTTS. The mean period of the transition disk candidates is $7.8 \pm 1.0$ days but the sample of periodic transition disk candidates is small compared to the diskless and full disk samples.

Following the light curve morphological classification from Alencar et al. (2010), the CoRoT light curves of the CTTS were classified by Sousa et al. (2016), as spot-like (sinusoidal variations due stable spots at the stellar surface), AA Tau-like (quasiperiodical eclipse like light curves explained as occultation of the stellar photosphere by an inner disk warp (see Alencar et al. 2010; Cody et al. 2014; Fonseca et al. 2014; McGinnis et al. 2015, for more details about AA Tau-like light curves), and non-periodic light curves, which includes non-periodic occultations of the stellar photosphere by the inner disk, accretion bursts (Stauffer et al. 2014), and variations not associated with an unique physical phenomenon.

We classified all the 2011 CoRoT WTTS light curve using the same nomenclature, and we adopted the morphological 


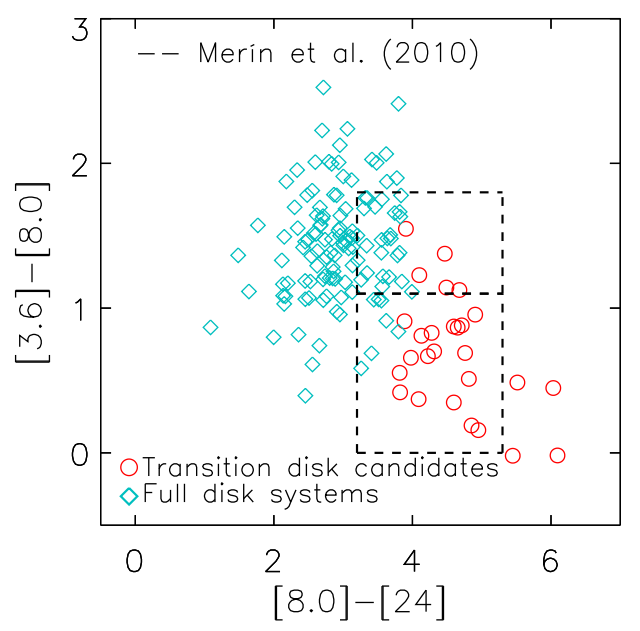

(a)

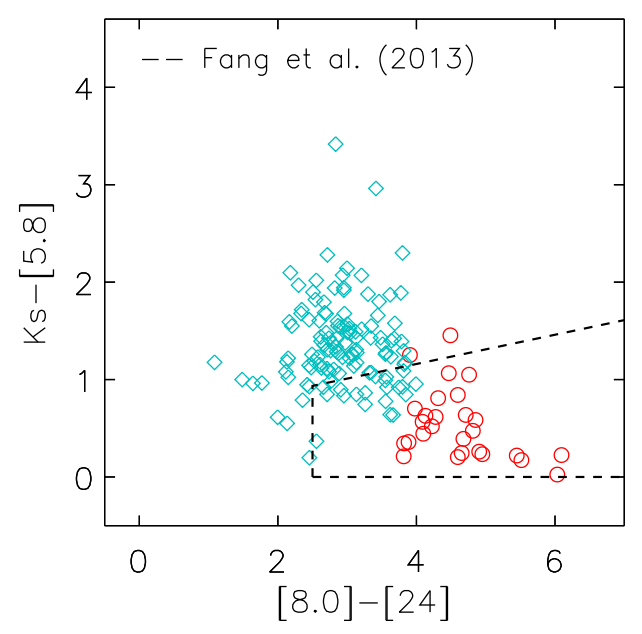

(c)

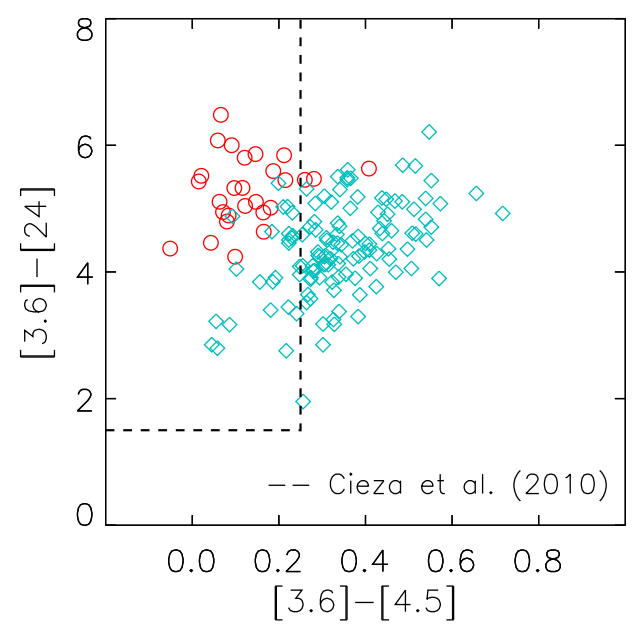

(b)

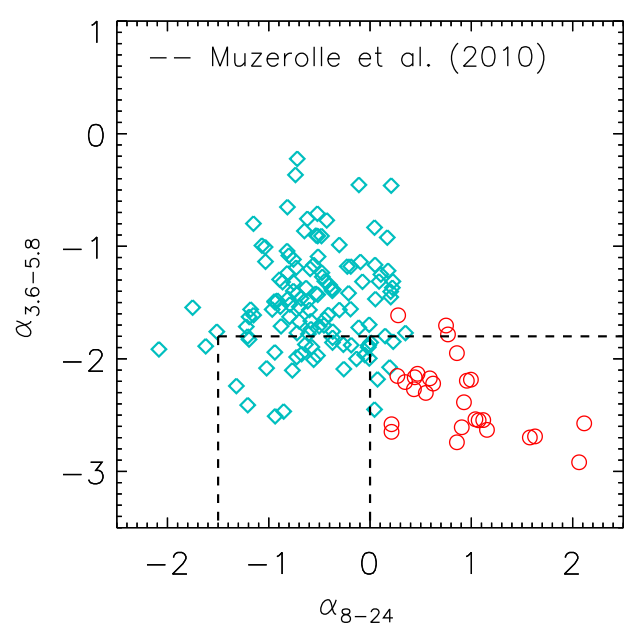

(d)
Fig. 5. Different diagrams that are used in previous works to separate transition disk systems from the full disk sample. (a) Selection criteria that were used by Merín et al. (2010). Systems that fall in the bottom dashed box are classified as transition disks and systems that fall in the top dashed box are systems with small excess in the IRAC bands. (b) Selection criteria used by Cieza et al. (2010). The dashed lines separate the transition disks. (c) Selection criteria from Fang et al. (2013). Systems that fall in the dashed region are transition disks. $(d)$ Selection criteria from Muzerolle et al. (2010). Systems in the left dashed box are classified as weak-excess sources and systems that lie in the right dashed box are transition disks. Almost all of our transition disk candidates fulfill the selection criteria from the literature.

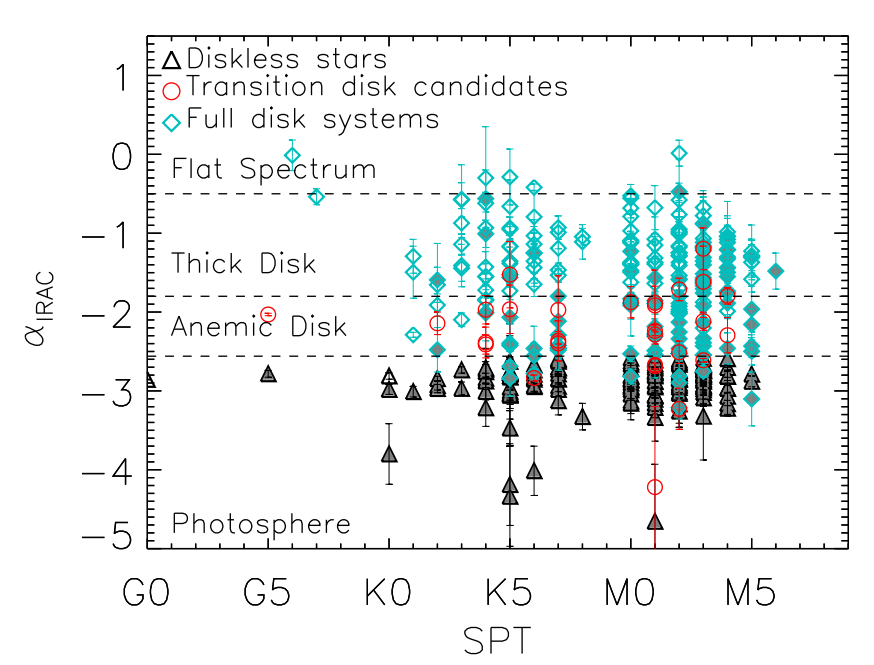

Fig. 6. Slope of the SED from $3.6 \mu \mathrm{m}$ to $8 \mu \mathrm{m}\left(\alpha_{\text {IRAC }}\right)$ measured by Teixeira et al. (2012) as a function of the spectral type obtained by Venuti et al. (2014), Dahm \& Simon (2005), Rebull et al. (2002), Walker (1956). Symbols indicate our categorization (notation as in Fig. 4). Our sample of transition disk candidates presents dust in the inner disk similar to anemic disks, which shows that anemic disk systems can be good candidates to transition disks. Systems for which we do not have data at $22 / 24 \mu \mathrm{m}$ are identified by gray filled symbols. classification of the CTTS from Sousa et al. (2016). The distribution of morphological classifications of the CoRoT light curve is shown in Fig. 12. Almost all $(\sim 78 \%)$ of the diskless stars are classified as spot-like. As the light curve morphology is related to system evolution (Sousa et al. 2016), diskless systems, which are no longer accreting, are expected to present spot-like light curves, as their photometric variability is mainly due to the cold surface spots.

Full disk systems are mostly CTTS and their light curve variability can be due to different physical processes, like accretion, circumstellar occultation, and spots. They present a variety of light curve morphologies, $\sim 38 \%$ being classified as non-periodic, probably the result of many physical phenomena acting together (accretion and spots, for example). The sample of transition disk candidates observed by CoRoT is small, but as most of them are also CTTS, we would then expect a light curve morphology similar to the full disk systems. It is interesting to note that three transition disk candidates are classified as AA Tau-like systems and two as due to aperiodic extinction. Extinction systems (periodic or not) are known to have dust in the inner disk (Alencar et al. 2010; Cody et al. 2014; Fonseca et al. 2014; McGinnis et al. 2015; Sousa et al. 2016), which is responsible for the occultation of the stellar photosphere, and they are common among the anemic disks (Alencar et al. 2010; Sousa et al. 2016). 


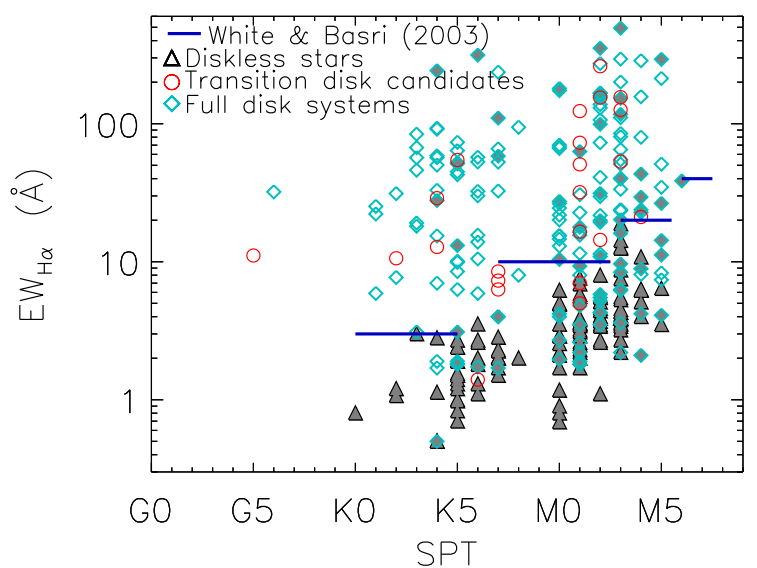

(a)

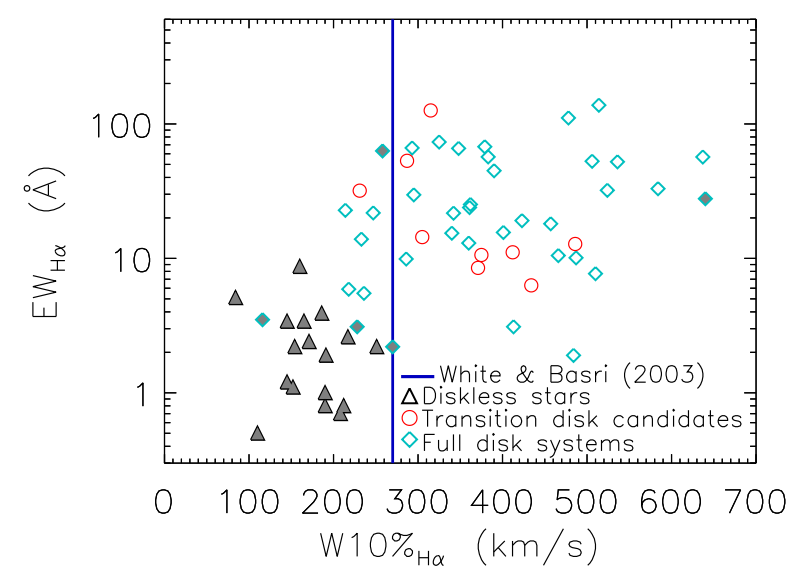

(b)

Fig. 7. $\mathrm{H} \alpha$ criteria (blue lines) to select CTTS and WTTS (White \& Basri 2003). (a) H $\alpha$ equivalent width vs. spectral type. (b) H $\alpha$ equivalent width vs. $\mathrm{H} \alpha$ width at $10 \%$ of maximum intensity. Transition disk candidates are predominantly found among accreting systems. Therefore, the presence of a hole in the inner disk does not stop the accretion process, suggesting that gas is flowing through the hole. Systems for which we do not have data at $22 / 24 \mu \mathrm{m}$ are identified by gray filled symbols.

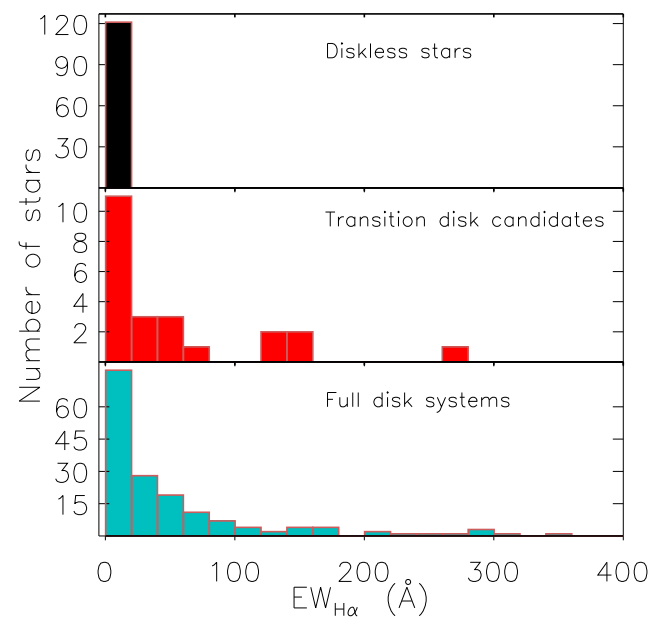

(a)

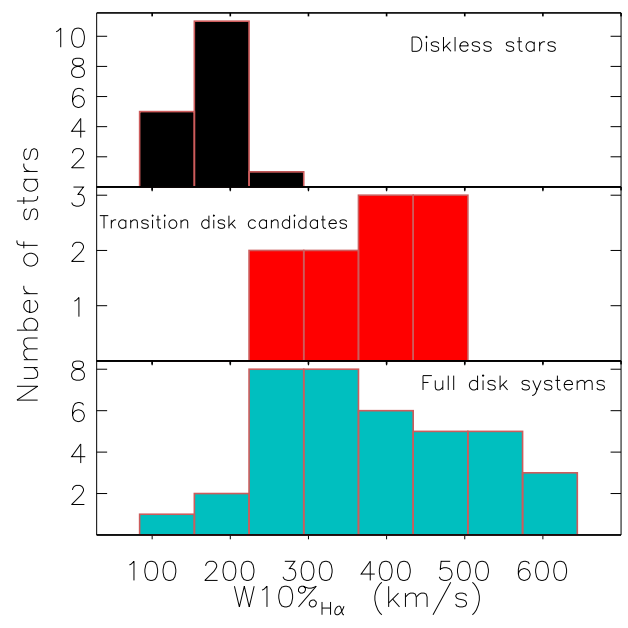

(b)
Fig. 8. Distribution of $\mathrm{H} \alpha$ emission line parameters. (a) $\mathrm{H} \alpha$ equivalent width and $(b) \mathrm{H} \alpha$ width at $10 \%$ of maximum intensity of our sample of diskless stars (black), transition disk candidates (red) and full disk systems (light blue). Transition disk candidates distributions are more similar to the full disk systems than diskless stars, see the text.

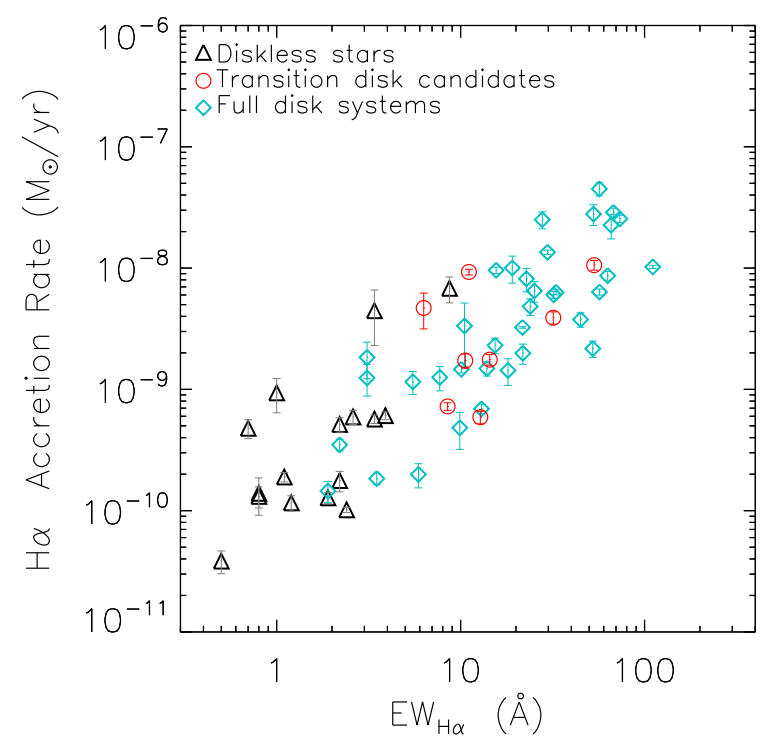

Fig. 9. Mass accretion rates from $\mathrm{H} \alpha$ equivalent width calculated by Sousa et al. (2016) as a function of $\mathrm{H} \alpha$ equivalent width. The error bar represents the night-to-night variability of the accretion rate.

\subsection{Transition disk candidates in NGC 2264 from the literature}

Systems with near-IR deficits and mid to far IR emission excess were initially associated with an inner disk hole by Strom et al. (1989), studying star-disk systems in the Taurus-Auriga star formation complex. Over the years, many new objects with inner holes were identified and have been studied in detail (Calvet et al. 2005; Najita et al. 2007; Espaillat et al. 2008; Muzerolle et al. 2010; Andrews et al. 2011; Manara et al. 2014; Bustamante et al. 2015).

Systems with inner disk holes have been identified in NGC 2264 by Sung et al. (2009), using Spitzer IRAC and MIPS data. The observed star-disk systems, in Sung et al. (2009), were classified as pre-transition and transition disks, following Espaillat et al. (2008). If the SED flux at $8.0 \mu \mathrm{m}$ was smaller than the flux at $24 \mu \mathrm{m}$, and if the sign of $\alpha_{\text {IRAC }}$ and $\alpha_{\text {IRAC-MIPS }}$ (the SED slope between $8.0 \mu \mathrm{m}$ and $24 \mu \mathrm{m}$ ) was different, the system was considered a candidate to have a hole/gap in the disk. Additionally, these systems were classified as pre-transition disks if $-0.3 \geq \alpha_{\text {IRAC }} \geq-1.8$ and as a transition disk if $\alpha_{\text {IRAC }}<-1.8$ (Sung et al. 2009).

Using these selection criteria, Sung et al. (2009) found 13 systems with pre-transition disks and 24 systems with 


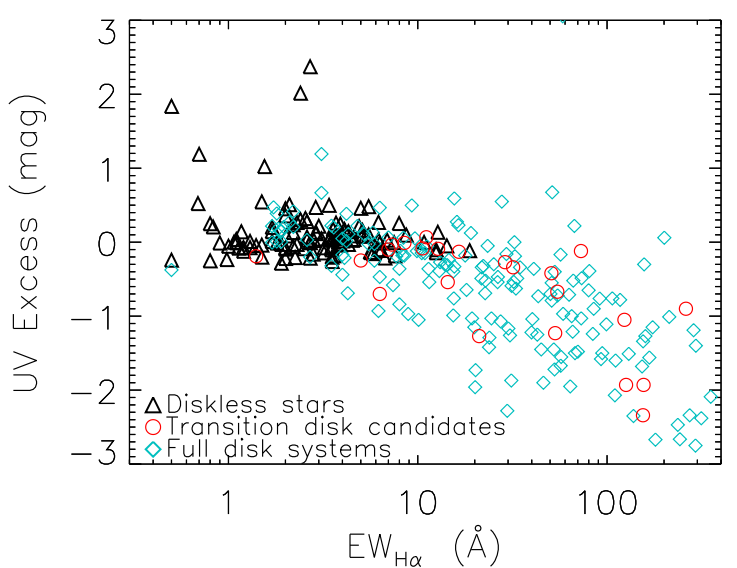

(a)

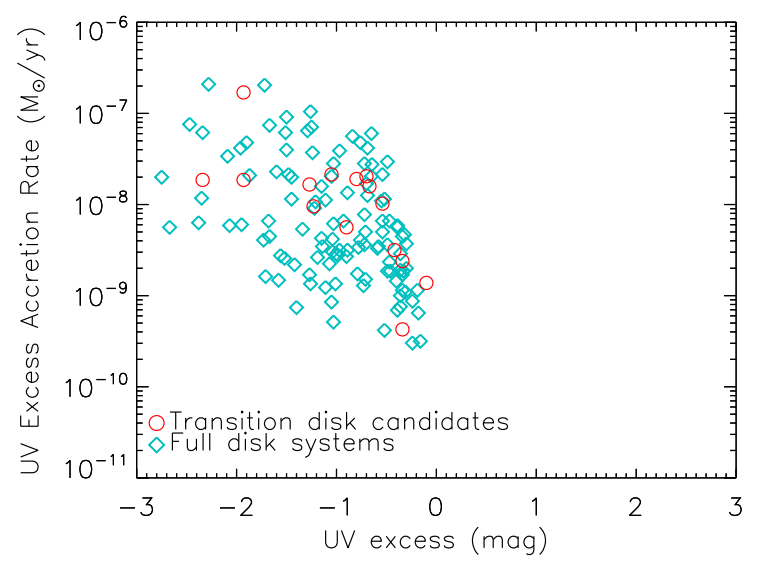

(b)

Fig. 10. UV excess as a function of $\mathrm{H} \alpha$ equivalent width $(a)$ and mass accretion rate as a function of UV excess $(b)$. More negative values indicate larger UV excess. The UV excess and mass accretion rate were calculated by Venuti et al. (2014) and the H $\alpha$ equivalent width was measured by Sousa et al. (2016) and Dahm \& Simon (2005). As expected, diskless stars do not present UV excess, while full disk systems do. Transition disk candidates, in general, present UV excess similar to stars with full disks, which is consistent with active accretion, as discussed in the text.

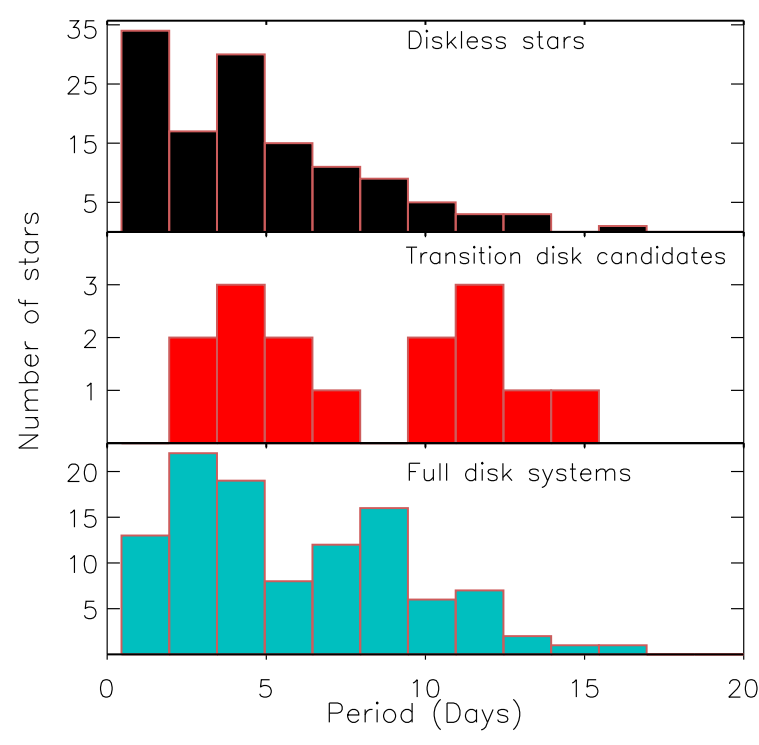

Fig. 11. Period distribution of the samples of diskless stars (black), transition disk candidates (red), and full disk systems (light blue), that were analyzed in this work. Diskless stars are substantially more rapid rotators than full disk systems, as expected, since most stars with full disks are CTTS and most diskless stars are WTTS.

transition disks belonging to NGC 2264. Among these 37 systems found by Sung et al. (2009), only 18 are part of the sample of stars analyzed in this work and only one of them was not classified as a transition disk candidate by us. We did not separate transition and pre-transition disks in our sample, and two systems (Mon-000177 and Mon-000961) of our transition disk candidates were classified as pre-transition disk by Sung et al. (2009).

Teixeira et al. (2012) found only three transition disks in NGC 2264. Their selection criteria were very strict and classified as transition disks only accreting systems that did not present dust in the inner disk (photospheres according to the $\alpha_{\text {IRAC }}$ classification) and had excess at $24 \mu \mathrm{m}$, indicating the existence of a thick outer disk. In our sample, we have only one star classified as a photosphere that has available data at $24 \mu \mathrm{m}$. This star accrete but does not present signs of an outer thick disk.

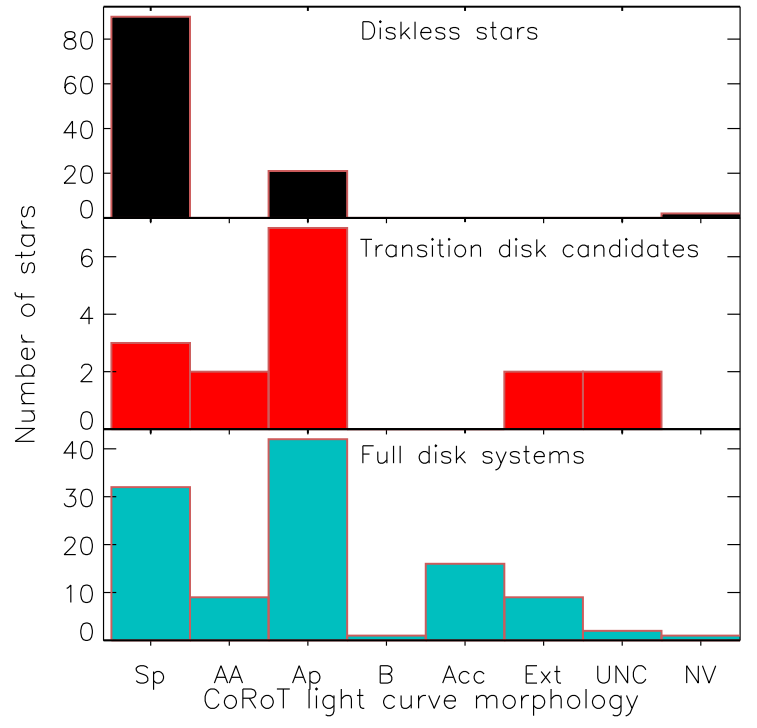

Fig. 12. Distribution of morphological classification of 2011 CoRoT light curve of our sample of TTS. The CTTS morphological classification was taken from Sousa et al. (2016), while the WTTS morphological classification is from this work. "SP" corresponds to spot-like, "AA" to AA Tau-like, "Ap" to aperiodic variations not related to a unique physical phenomenon, "B" to eclipsing binary, "Acc" to accretion bursts, "Ext" to non-periodic extinction, "UNC" to unclassified light curves, and "NV" to non-variable light curves.

Rapson et al. (2014) also identified transition disks in their sample of stars from NGC 2264 observed with the Spitzer satellite. Their selection was based on photometric criteria. They classified as transition disks those Class III systems that have [5.8] - [24] > 2.5 mag or [4.5] - [24] $>2.5 \mathrm{mag}$ and [3.6] $<$ $14 \mathrm{mag}$, which are systems that have excess at $24 \mu \mathrm{m}$. They classified 44 systems as transition disks using these criteria, but only 26 are part of our sample. Among the 26 systems we have in common, our classification agrees with theirs for 17 transition disk candidates, while the other 9 systems were classified by us as full disks.

In this work we found seven new transition disk candidates belonging to NGC 2264 that had not been classified as transition disks before. These systems were classified as Class II or III by 
Table 4. Parameters obtained by Hyperion SED fitting code for sample of transition disk candidates.

\begin{tabular}{|c|c|c|c|c|c|c|c|c|c|c|}
\hline Mon ID $^{(a)}$ & $A_{\mathrm{V}}{ }^{(b)}$ & $\begin{array}{r}\operatorname{Dist}^{(b)} \\
(\mathrm{pc})\end{array}$ & $\begin{array}{r}T_{\star}{ }^{(b)} \\
(\mathrm{K})\end{array}$ & $\begin{array}{r}R_{\star}(b) \\
\left(R_{\odot}\right)\end{array}$ & $\begin{array}{r}M_{\text {disk }}(b) \\
\left(M_{\odot}\right)\end{array}$ & $\begin{array}{r}R_{\mathrm{H}}{ }^{(c)} \\
(\mathrm{AU})\end{array}$ & $\begin{array}{l}R_{\mathrm{in}}(b) \\
\left(R_{\mathrm{Sub}}\right)\end{array}$ & $\begin{array}{r}R_{\mathrm{Sub}}(c) \\
(\mathrm{AU})\end{array}$ & $\begin{array}{r}R_{\operatorname{Max}}(b) \\
(\mathrm{AU})\end{array}$ & $\begin{array}{r}i^{(b)} \\
\left(^{\circ}\right) \\
\end{array}$ \\
\hline Mon-000040 ${ }^{(*)}$ & 0.1 & 698.2 & 2832.0 & 0.91 & 0.0005293 & 5.85 & 419.1 & 0.0140 & 372.7 & 77.2 \\
\hline Mon-000120 & 1.2 & 698.2 & 3775.0 & 1.84 & 0.0001934 & 0.53 & 10.4 & 0.0514 & 472.9 & 21.9 \\
\hline Mon-000122 $2^{(*)}$ & 0.8 & 693.4 & 4230.0 & 1.33 & 0.0008640 & 5.84 & 124.4 & 0.0469 & 112.0 & 76.7 \\
\hline Mon-000177 & 0.3 & 690.2 & 4590.0 & 2.66 & 0.002971 & 0.23 & 2.0 & 0.1114 & 951.0 & 55.2 \\
\hline Mon-000273 & 0.2 & 527.2 & 3738.0 & 1.31 & $1.844 \mathrm{E}-05$ & 17.85 & 498.1 & 0.0358 & 1314.0 & 68.1 \\
\hline Mon-000280 & 0.5 & 699.8 & 3922.0 & 1.33 & 0.006200 & 0.09 & 2.2 & 0.0401 & 61.1 & 14.0 \\
\hline Mon-000296 & 0.6 & 758.6 & 5214.0 & 1.60 & $8.402 \mathrm{E}-06$ & 0.12 & 1.4 & 0.0872 & 601.8 & 64.8 \\
\hline Mon-000314 & 0.3 & 701.5 & 2918.0 & 1.76 & 0.004796 & 3.58 & 125.1 & 0.0286 & 186.8 & 76.4 \\
\hline Mon-000328 & 0.2 & 753.4 & 3338.0 & 1.50 & $8.831 \mathrm{E}-07$ & 3.51 & 108.5 & 0.0324 & 1093.0 & 9.3 \\
\hline Mon-000342 ${ }^{(*)}$ & 0.3 & 916.2 & 3836.0 & 2.09 & $4.624 \mathrm{E}-08$ & 18.42 & 306.7 & 0.0601 & 117.9 & 82.4 \\
\hline Mon-000433 & 1.2 & 726.1 & 3506.0 & 2.46 & $2.443 \mathrm{E}-06$ & 7.39 & 126.0 & 0.0587 & 250.9 & 65.4 \\
\hline Mon-000452 (*) & 0.1 & 827.9 & 3605.0 & 2.35 & $3.533 \mathrm{E}-06$ & 6.00 & 101.0 & 0.0594 & 1796.0 & 86.2 \\
\hline Mon-000502 & 1.4 & 662.2 & 4263.0 & 1.74 & $1.757 \mathrm{E}-05$ & 9.43 & 150.7 & 0.0626 & 425.6 & 77.7 \\
\hline Mon-000637 & 1.1 & 756.8 & 3650.0 & 1.82 & 0.0005777 & 8.66 & 183.2 & 0.0473 & 1172.0 & 79.2 \\
\hline Mon-000676 & 0.3 & 712.9 & 3864.0 & 2.35 & $7.821 \mathrm{E}-05$ & 8.50 & 123.6 & 0.0688 & 66.9 & 75.7 \\
\hline Mon-000771 & 0.3 & 744.7 & 5243.0 & 2.02 & $1.408 \mathrm{E}-06$ & 77.93 & 696.8 & 0.1118 & 2331.0 & 83.7 \\
\hline Mon-000824 & 0.9 & 636.8 & 3864.0 & 2.35 & $7.821 \mathrm{E}-05$ & 8.50 & 123.6 & 0.0688 & 66.9 & 3.6 \\
\hline Mon-000860 & 0.4 & 690.2 & 3807.0 & 0.90 & 0.0001289 & 4.34 & 170.2 & 0.0255 & 53.9 & 49.2 \\
\hline Mon-000879 & 0.3 & 966.1 & 3864.0 & 2.35 & $7.821 \mathrm{E}-05$ & 8.50 & 123.6 & 0.0688 & 66.9 & 3.6 \\
\hline Mon-000937 ${ }^{(*)}$ & 0.8 & 948.4 & 3563.0 & 3.20 & $6.025 \mathrm{E}-08$ & 22.31 & 282.1 & 0.0791 & 314.0 & 72.1 \\
\hline Mon-000961 & 0.1 & 682.3 & 2723.0 & 1.15 & 0.0003476 & 0.28 & 17.1 & 0.0162 & 2359.0 & 56.0 \\
\hline Mon-000965 & 0.1 & 803.5 & 3028.0 & 2.05 & 0.001175 & 9.50 & 263.3 & 0.0361 & 1448.0 & 6.4 \\
\hline Mon-000997 ${ }^{(*)}$ & 0.8 & 663.7 & 3374.0 & 1.00 & $2.228 \mathrm{E}-08$ & 2.61 & 118.5 & 0.0220 & 270.4 & 57.9 \\
\hline Mon-001033 ${ }^{(*)}$ & 0.5 & 756.8 & 3999.0 & 1.70 & 0.0009192 & 16.66 & 312.0 & 0.0534 & 221.4 & 65.8 \\
\hline Mon-001094 & 0.3 & 727.8 & 3948.0 & 1.70 & $2.806 \mathrm{E}-07$ & 21.66 & 416.8 & 0.0520 & 112.7 & 19.8 \\
\hline Mon-001229 & 1.3 & 798.0 & 4063.0 & 1.41 & 0.0006760 & 0.59 & 12.9 & 0.0459 & 120.1 & 10.1 \\
\hline Mon-001287 & 1.9 & 814.7 & 3995.0 & 1.06 & $8.285 \mathrm{E}-08$ & 2.64 & 79.8 & 0.0331 & 164.0 & 12.6 \\
\hline Mon-001308 & 0.3 & 774.5 & 5011.0 & 2.13 & $1.981 \mathrm{E}-06$ & 20.18 & 188.7 & 0.1069 & 298.1 & 86.4 \\
\hline
\end{tabular}

Notes. This table is ordered according to the Mon ID. ${ }^{(a)}$ CSIMon is an internal identification of the CSI 2264 campaign. Here, CSI was omitted for brevity. ${ }^{(b)}$ The model parameters of the best SED fitting: reddening $\left(A_{\mathrm{v}}\right)$, distance of the star to the Sun (Dist), central star temperature $\left(T_{\star}\right)$ and radius $\left(R_{\star}\right)$, disk mass $\left(M_{\text {disk }}\right)$, inner disk radius $\left(R_{\text {in }}\right)$, outer disk radius $\left(R_{\text {Max }}\right)$ and inclination of the system $(i) .{ }^{(c)}$ Parameters determined using parameters from SED fitting model. ${ }^{(*)}$ New transition disk candidates identified in this work.

Sung et al. (2009) and Rapson et al. (2014). We identified these systems in Table 4 with an asterisk after the CSIMon ID.

\subsection{Disk and inner hole characteristics}

Disk parameters were computed by Hyperion SED fitting code. The size of the inner disk hole corresponds to the inner radius of the disk $\left(R_{\text {in }}\right)$, which is an output parameter of Hyperion SED fitting code (Robitaille 2017). As explained in Sect. $3, R_{\text {in }}$ is the dust sublimation radius for model 2 , that is composed of a star and a disk. In model 3 , that includes a star and a disk with an inner disk hole, $R_{\text {in }}$, that can vary from 1 to $1000 R_{\text {sub. To determine the }}$ sublimation radius, we used the empirical equation obtained by Whitney et al. (2004) for a fixed type of dust:

$\frac{R_{\mathrm{Sub}}}{R_{\star}}=\left(\frac{T_{\mathrm{Sub}}}{T_{\star}}\right)^{-2.085}$,

where $T_{\text {Sub }}$ is the dust sublimation temperature $(1600 \mathrm{~K})$ and $R_{\star}$ and $T_{\star}$ are the radius and effective temperature of the central star, respectively. In Fig. 13, we show the distributions of dust disk mass, the inner disk radii, and the external disk radii. The disk mass and the external disk radii are however only estimated values and correspond to lower limits, since we do not have broad wavelength coverage.
The size of the inner disk hole $\left(R_{\mathrm{H}}\right)$ is given by the inner disk radius when it is larger than the sublimation radius. We calculated $R_{\mathrm{H}}$ for all transition disk candidates. Then we compared the inner disk hole size with different characteristics of the star-disk system; see Figs. 14 and 15. In Table 4, we show the parameters obtained by Hyperion SED fitting code for each transition disk candidate and the hole size inferred from the best model parameters.

In our sample of transition disk candidates, the hole size varies from 0.09 to $78 \mathrm{AU}$, with a mean value of $10.4 \pm 2.8 \mathrm{AU}$. These estimated hole sizes are smaller than the values usually found in the literature in other star forming regions. Normally there is a considerable population with hole sizes larger than $10 \mathrm{AU}$, as seen, for example, in Ercolano \& Pascucci (2017). However, the largest hole sizes from models, found in the literature, were obtained by SED fitting with additional mid and far IR observational data (e.g., van der Marel et al. 2016). As discussed in Merín et al. (2010), systems with larger hole sizes are also more easily found with IR spectra than photometric data. The other possibility is that the SED model used in this work, which has only a passive disk and does not take into account the heating of the disk due to accretion, tends to produce smaller disk holes, as reported by Merín et al. (2010).

Different mechanisms can be responsible for creating a hole in the inner disk. Photoevaporation of the inner disk by stellar 

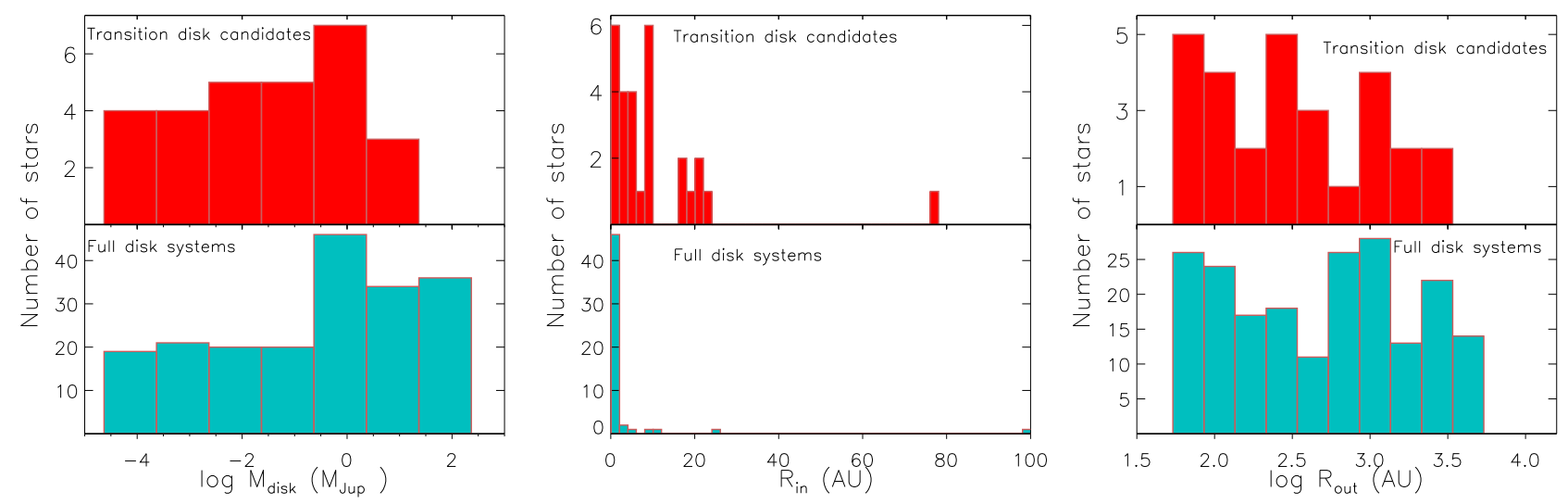

Fig. 13. Distribution of disk parameters obtained by the SED model fitting of transition disk and full disk systems. Left: disk dust mass. Middle: disk inner radius. Right: disk outer radius. For the transition disk sample, the disk inner radius is the inner hole size and for the sample of full disk systems it corresponds to the dust sublimation radius. As the transition disk systems have a disk inner hole, the inner disk radius of the transition disk systems tends to be larger than the full disk systems. The disk mass and the external disk radii are however only estimated values and correspond to lower limits, see text.

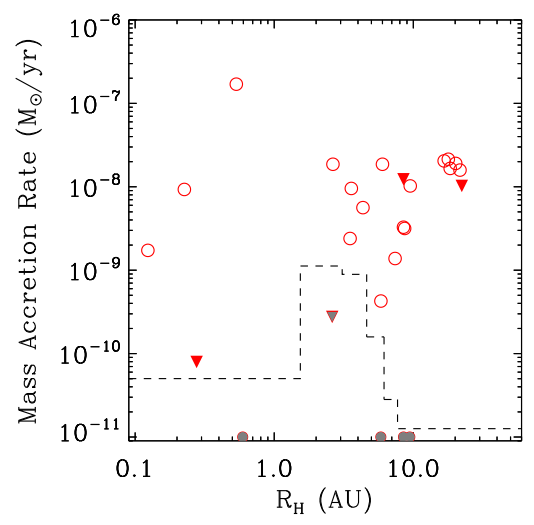

(a)

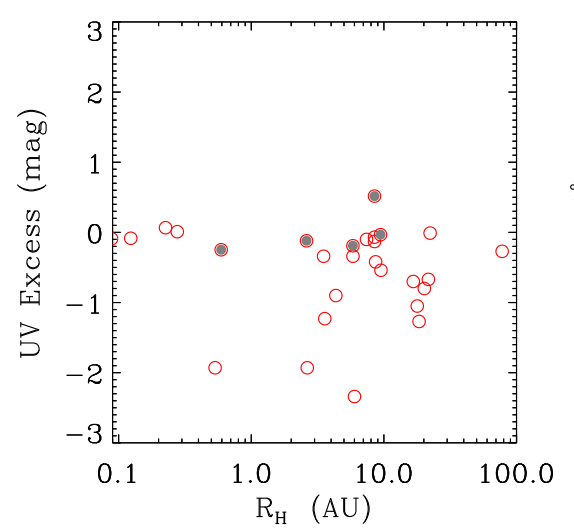

(b)

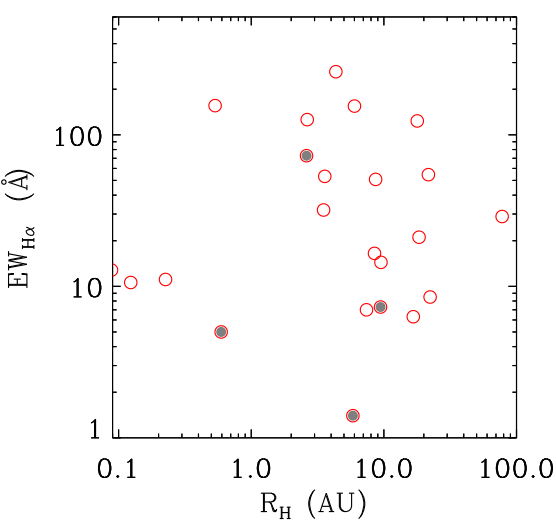

(c)

Fig. 14. Accretion diagnostic as a function of disk hole size for transition disk candidates. (a) Mass accretion rates are from UV excess (preferentially) (Venuti et al. 2014) and from $\mathrm{H} \alpha$ equivalent width (Sousa et al. 2016). For WTTS, we fix the value of the mass accretion rate at $1 \times 10^{-11} M_{\odot} \mathrm{yr}^{-1}$. The upside down triangles correspond to the upper limits of the mass accretion rate. The dashed line represents the region where the inner hole can be explained by X-ray photoevaporation (Owen et al. 2011, 2017). Only 18\% of our sample of transition disk candidates can be explained by X-ray photoevaporation of the inner disk by stellar radiation. (b) UV emission excess (Venuti et al. 2014). (c) H $\alpha$ equivalent width (Sousa et al. 2016; Dahm \& Simon 2005). Gray filled symbols identify systems that fall in the region where the inner disk hole can be explained by X-ray photoevaporation.

radiation can open holes, but, in general, with small radii $\left(R_{\mathrm{H}}<\right.$ $10 \mathrm{AU})$ and for small mass accretion rates $\left(\lesssim 10^{-9} M_{\odot} \mathrm{yr}^{-1}\right)$ (Owen et al. 2011; Manara et al. 2014; Owen 2016). Photoevaporation is actually more efficient in non-accreting systems, opening large holes in the disk ( $\gtrsim 20 \mathrm{AU}$ ) (Owen 2016).

In Fig. 14a we plot the mass accretion rate as a function of the hole size and identify the region where the inner hole can be due to photoevaporation alone, according to the model by Owen et al. (2011, 2017). As the WTTS do not accrete, we fix the mass accretion rate at $1 \times 10^{-11} M_{\odot} \mathrm{yr}^{-1}$ for these systems to show the position of these systems on the plot. We indicated the five systems that fall in the photoevaporation region in Fig. 14. We can see that almost all our transition disk candidates are outside of the region where photoevaporation can explain the inner disk hole. Our sample of non-accreting transition disks is small, and the four systems also present small inner holes $\left(R_{\mathrm{H}}<10 \mathrm{AU}\right)$. Observed non-accreting transition disks with the largest holes are indeed rare (Owen 2016).

To analyze the relation of the hole size with the accretion process, we also plot the UV excess (Fig. 14b) and the H $\alpha$ equiv- alent width (Fig. 14c) as a function of the hole size. Transition disk systems that present UV excesses (more negative values in the plot) tend to have larger holes compared to transition disk systems without UV excesses.

We analyzed the relation of the hole size with the IR emission in the inner and outer disk and with the $\alpha_{\text {IRAC }}$ index (Fig. 15). Transition disk candidates with lower emission in the inner disk tend to have large holes (Fig. 15a). Our data do not show a relation between the hole size and the mid-IR emission and the $\alpha_{\mathrm{IRAC}}$ index (Figs. 15b and c).

Planet formation in the disk is one of the most plausible mechanisms to explain transition disk systems that present signs of accretion, despite the fact that the models do not explain all the observational characteristics of transition disks (Owen 2016; Ercolano \& Pascucci 2017; Zhu et al. 2011). Considering the evolution sequence of a disk with a planet, as discussed by Owen (2016), when a newly formed planet is large enough to open a gap in the disk, it effectively traps the mm dust in the outer disk. At this point, the inner disk is still not dust free, and the SED looks like a full disk. After that, due to some mechanism 


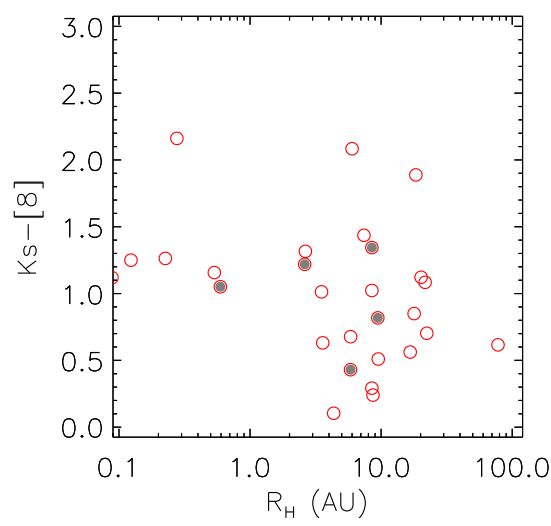

(a)

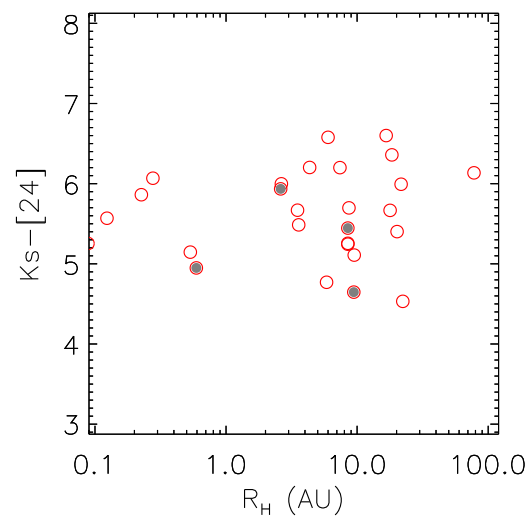

(b)

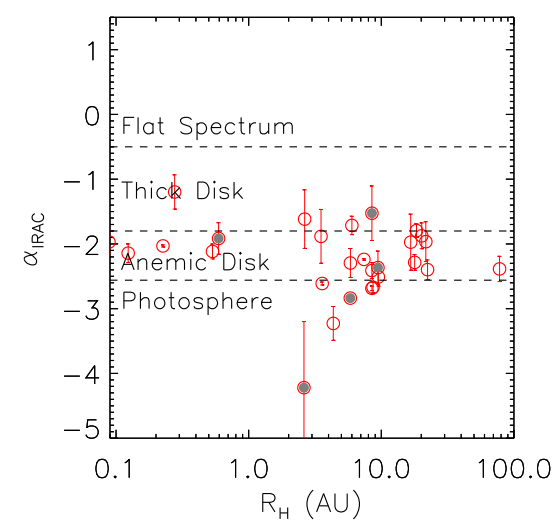

(c)

Fig. 15. Disk diagnostics as a function of disk hole size for our sample of transition disk candidates. (a) Near-IR color, $(b)$ mid-IR color, and (c) $\alpha_{\text {IRAC }}$ index. Gray filled symbols identify systems that fall in the region where the inner disk hole can be explained by X-ray photoevaporation.

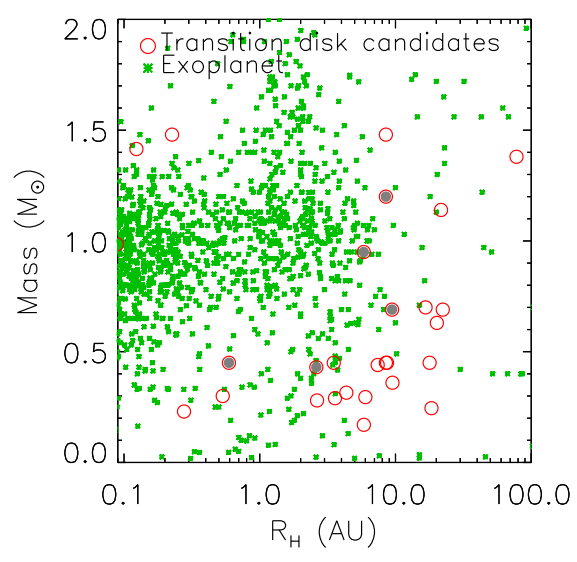

(a)

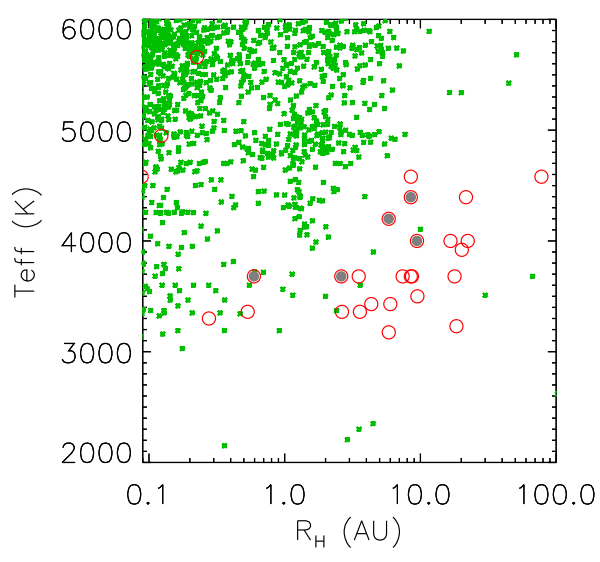

(b)

Fig. 16. Mass and temperature of the central star obtained by Venuti et al. (2014) as a function of disk hole size. The overplotted data from the literature correspond to exoplanets with confirmed detection and the vertical axis is plotted as a function of the semimajor axis of the exoplanet orbit. Despite the fact that the masses and temperatures of our stars are slightly lower than the exoplanet host stars, the hole sizes of our transition disks and the semimajor axis of the exoplanet orbits are compatible. The data of the exoplanets and the host star were taken from http:// exoplanet. eu. Gray filled symbols identify systems that fall in the region where the inner disk hole can be explained by X-ray photoevaporation. Our data do not show a clear linear tendency between the mass and the temperature of central star with the inner hole size, as seen in literature.

that is not well understood, the inner disk turns almost dust free and the SED is consequently modeled as a disk with an inner hole, that correspond to an accreting transition disk system characterized to be mm-bright. During this phase, the young planet can then migrate to orbits with smaller radii ( $\lesssim 10 \mathrm{AU})$. Consequently the dust from the outer disk can move to inner orbits due to disk viscosity, and the inner disk can be photoevaporated by radiation of the central star, forming non-accreting transition disks with small holes and bright in the $\mathrm{mm}$ or systems with a gap formed by photoevaporation of the outer disk. The current disk models with a planet predict more non-accreting systems with large holes, and accreting systems with small holes and mm-bright, than found observationally (e.g., Owen 2016) and also confirmed with our sample (see Fig. 14a). The systems that fall out of the region limited by the dashed lines in Fig. 14a are good candidates to have proto-planets in different stages. Exploring and modeling these systems using data from wavelengths long than $24 \mu \mathrm{m}$ is beyond the scope of this work.

In Fig. 16 we show the mass and temperature of the central star (obtained by Venuti et al. 2014) as a function of the disk hole size for our transition disk candidates. Kim et al. (2009) found a linear relation between the size of the disk hole and the mass and temperature of the central star for a sample of transition disks belonging to Chamaeleon I, indicating the dependence of the hole size with the central star's properties. In our sample of transition disk candidates, this tendency is not well defined in the plot. In Fig. 16, we also overplotted literature data of semimajor axis of the orbit of confirmed exoplanets and their host star masses. Despite the fact that the masses and temperatures of our stars are slightly lower than the exoplanet host stars, the hole sizes $\left(R_{\mathrm{H}}\right)$ of our transition disks and the semimajor axis of the exoplanet orbits are compatible. This result is intuitively expected, if the planets were responsible for opening the gap in the inner disk.

\section{Conclusions}

In this work we searched for transition disk candidates in the young cluster NGC 2264. Our sample of 401 TTS was observed with Spitzer equipped with IRAC instrument and MegaCam on CFHT, and corresponds to the star-disk systems with well defined stellar, disk, and accretion parameters. The main results of this work are described below. 
We modeled the SEDs of 401 TTS with the Hyperion SED fitting code, using three different sets of models that correspond to a single stellar photosphere, a star with a full passive disk and a star with a passive disk and an inner disk hole. With the results of the model, we separated the star-disk systems into full disk systems (209 systems), diskless stars (164 systems), and transition disk candidates ( 28 systems). The transition disk candidates represent $7 \%$ of our sample and confirm that transition disks are a rapid phase of disk evolution.

We have shown that transition disk systems present $\mathrm{H} \alpha$, UV excess, and mass accretion rates at the same level as accreting full disk systems. It shows that the presence of a hole in the inner disk does not stop the accretion process, since $\sim 82 \%$ of our transition disk candidates still accrete, suggesting that gas is flowing through the disk hole, as found in transition disks from other star formation regions.

Our sample of transition disk candidates have dust in the inner disk similar to anemic disks, which indicates that anemic disks can be in general good candidates to have transition disks.

In color-color diagrams, we see three different populations. Stars with a full disk present IR excess above the photospheric emission, in the inner and outer parts of the disk. The transition disk systems present weak dust emission in the inner disk, like diskless stars, and dust emission in the outer disk like full disk systems, which is consistent with disk evolution from inside to outside, associated with photoevaporation of the inner disk by the stellar radiation or planet formation.

We estimated the hole size of our sample of transition disk candidates and found sizes from 0.09 to $78 \mathrm{AU}$ with a mean value of $10.4 \pm 2.8 \mathrm{AU}$. Among our sample of transition disk candidates, only $\sim 18 \%$ have small mass accretion rates $\left(\lesssim 10^{-9} M_{\odot} \mathrm{yr}^{-1}\right)$ and small hole sizes $\left(R_{\mathrm{H}}<10 \mathrm{AU}\right)$ that can be explained by X-ray photoevaporation of the inner disk by stellar radiation. We also show that $\sim 82 \%$ could be explained by planet formation in different evolutionary stages (with small and large hole sizes).

Acknowledgements. APS and SHPA acknowledge support from CNPq, CAPES and Fapemig. We also thank Laura Venuti and Julia Roquette for helpful discussions. This publication makes use of data products from the Two Micron All Sky Survey, which is a joint project of the University of Massachusetts and the Infrared Processing and Analysis Center/California Institute of Technology, funded by the National Aeronautics and Space Administration and the National Science Foundation. This work has made use of data from the European Space Agency (ESA) mission Gaia (https://www.cosmos.esa.int/gaia), processed by the Gaia Data Processing and Analysis Consortium (DPAC, https://www. cosmos.esa.int/web/gaia/dpac/consortium). Funding for the DPAC has been provided by national institutions, in particular the institutions participating in the Gaia Multilateral Agreement. This work is based in part on observations made with the Spitzer Space Telescope, which is operated by the Jet Propulsion Laboratory, California Institute of Technology under a contract with NASA. This publication makes use of data products from the Wide-field Infrared Survey Explorer, which is a joint project of the University of California, Los Angeles, and the Jet Propulsion Laboratory/California Institute of Technology, funded by the National Aeronautics and Space Administration. Funding for SDSS-III has been provided by the Alfred P. Sloan Foundation, the Participating Institutions, the National Science Foundation, and the U.S. Department of Energy Office of Science. The SDSS-III web site is http://www. sdss3.org. SDSS-III is managed by the Astrophysical Research Consortium for the Participating Institutions of the SDSS-III Collaboration including the University of Arizona, the Brazilian Participation Group, Brookhaven National Laboratory, Carnegie Mellon University, University of Florida, the French Participation Group, the German Participation Group, Harvard University, the Instituto de Astrofisica de Canarias, the Michigan State/Notre Dame/JINA Participation Group, Johns Hopkins University, Lawrence Berkeley National Laboratory, Max Planck Institute for Astrophysics, Max Planck Institute for Extraterrestria Physics, New Mexico State University, New York University, Ohio State University, Pennsylvania State University, University of Portsmouth, Princeton University, the Spanish Participation Group, University of Tokyo, University of Utah,
Vanderbilt University, University of Virginia, University of Washington, and Yale University.

\section{References}

Adams, F. C., Hollenbach, D., Laughlin, G., \& Gorti, U. 2004, ApJ, 611, 360 Affer, L., Micela, G., Favata, F., Flaccomio, E., \& Bouvier, J. 2013, MNRAS, 430, 1433

Alencar, S. H. P., Teixeira, P. S., Guimarães, M. M., et al. 2010, A\&A, 519, A88 Alexander, R., Pascucci, I., Andrews, S., Armitage, P., \& Cieza, L. 2014, Protostars and Planets VI, 475

Andrews, S. M., Wilner, D. J., Espaillat, C., et al. 2011, ApJ, 732, 42

Andrews, S. M., Wilner, D. J., Zhu, Z., et al. 2016, ApJ, 820, L40

Andrews, S. M., Huang, J., Pérez, L. M., et al. 2018, ApJ, 869, L41

Ansdell, M., Williams, J. P., van der Marel, N., et al. 2016, ApJ, 828, 46

Bouvier, J., Cabrit, S., Fernandez, M., Martin, E. L., \& Matthews, J. M. 1993, A\&A, 272, 176

Bustamante, I., Merín, B., Ribas, Á., et al. 2015, A\&A, 578, A23

Calvet, N., \& Gullbring, E. 1998, ApJ, 509, 802

Calvet, N., D'Alessio, P., Hartmann, L., et al. 2002, ApJ, 568, 1008

Calvet, N., D’Alessio, P., Watson, D. M., et al. 2005, ApJ, 630, L185

Cieza, L., Padgett, D. L., Stapelfeldt, K. R., et al. 2007, ApJ, 667, 308

Cieza, L. A., Schreiber, M. R., Romero, G. A., et al. 2010, ApJ, 712, 925

Clarke, C. J., Gendrin, A., \& Sotomayor, M. 2001, MNRAS, 328, 485

Cody, A. M., Stauffer, J. R., Micela, G., Baglin, A., \& CSI 2264 Team 2013, Astron. Nachr., 334, 63

Cody, A. M., Stauffer, J., Baglin, A., et al. 2014, AJ, 147, 82

Dahm, S. E., \& Simon, T. 2005, AJ, 129, 829

Dullemond, C. P., \& Dominik, C. 2005, A\&A, 434, 971

Ercolano, B., \& Pascucci, I. 2017, R. Soc. Open Sci., 4, 170114

Espaillat, C., Calvet, N., Luhman, K. L., Muzerolle, J., \& D'Alessio, P. 2008, ApJ, 682, L125

Espaillat, C., Ingleby, L., Hernández, J., et al. 2012, ApJ, 747, 103

Espaillat, C., Muzerolle, J., Najita, J., et al. 2014, Protostars and Planets VI, 497

Fang, M., van Boekel, R., Wang, W., et al. 2009, A\&A, 504, 461

Fang, M., Kim, J. S., van Boekel, R., et al. 2013, ApJS, 207, 5

Fazio, G. G., Hora, J. L., Allen, L. E., et al. 2004, ApJS, 154, 10

Fitzpatrick, E. L. 1999, PASP, 111, 63

Flaccomio, E., Micela, G., \& Sciortino, S. 2006, A\&A, 455, 903

Fonseca, N. N. J., Alencar, S. H. P., Bouvier, J., Favata, F., \& Flaccomio, E. 2014,

\section{A\&A, 567, A39}

Gaia Collaboration (Prusti, T., et al.) 2016, A\&A, 595, A1

Gaia Collaboration (Brown, A. G. A., et al.) 2018, A\&A, 616, A1

Garaud, P., Meru, F., Galvagni, M., \& Olczak, C. 2013, ApJ, 764, 146

Grant, S. L., Espaillat, C. C., Megeath, S. T., et al. 2018, ApJ, 863, 13

Gunn, J. E., Carr, M., Rockosi, C., et al. 1998, AJ, 116, 3040

Haisch, Jr., K. E., Lada, E. A., \& Lada, C. J. 2001, ApJ, 553, L153

Hartmann, L., Calvet, N., Gullbring, E., \& D’Alessio, P. 1998, ApJ, 495, 385

Hernández, J., Calvet, N., Briceño, C., et al. 2007, ApJ, 671, 1784

Hollenbach, D., Gorti, U., Meyer, M., et al. 2005, ApJ, 631, 1180

Hughes, A. M., Andrews, S. M., Espaillat, C., et al. 2009, ApJ, 698, 131

Indebetouw, R., Mathis, J. S., Babler, B. L., et al. 2005, ApJ, 619, 931

Ingleby, L., Calvet, N., Bergin, E., et al. 2011, ApJ, 743, 105

Irwin, J., \& Bouvier, J. 2009, in IAU Symp., eds. E. E. Mamajek, D. R. Soderblom, \& R. F. G. Wyse, 258, 363

Kim, K. H., Watson, D. M., Manoj, P., et al. 2009, ApJ, 700, 1017

Kim, K. H., Watson, D. M., Manoj, P., et al. 2013, ApJ, 769, 149

Koepferl, C. M., Ercolano, B., Dale, J., et al. 2013, MNRAS, 428, 3327

Konigl, A. 1989, ApJ, 342, 208

Lada, C. 1987, Star Form. Reg., 115, 1

Lada, C. J., Muench, A. A., Luhman, K. L., et al. 2006, ApJ, 131, 1574

Lamm, M. H., Bailer-Jones, C. A. L., Mundt, R., Herbst, W., \& Scholz, A. 2004, A\&A, 417, 557

Lamm, M. H., Mundt, R., Bailer-Jones, C. A. L., \& Herbst, W. 2005, A\&A, 430, 1005

Luri, X., Brown, A. G. A., Sarro, L. M., et al. 2018, A\&A, 616, A9

Manara, C. F., Testi, L., Rigliaco, E., et al. 2013, A\&A, 551, A107

Manara, C. F., Testi, L., Natta, A., et al. 2014, A\&A, 568, A18

Marilli, E., Frasca, A., Covino, E., et al. 2007, A\&A, 463, 1081

McGinnis, P. T., Alencar, S. H. P., Guimarães, M. M., et al. 2015, A\&A, 577, A11

Merín, B., Brown, J. M., Oliveira, I., et al. 2010, ApJ, 718, 1200

Muzerolle, J., Allen, L. E., Megeath, S. T., Hernández, J., \& Gutermuth, R. A. 2010, ApJ, 708, 1107

Najita, J. R., Strom, S. E., \& Muzerolle, J. 2007, MNRAS, 378, 369

Ormel, C. W., Spaans, M., \& Tielens, A. G. G. M. 2007, A\&A, 461, 215 
A. P. Sousa et al.: A study of accretion and disk diagnostics in the NGC 2264 cluster

Owen, J. E. 2016, PASA, 33, e005

Owen, J. E., Ercolano, B., \& Clarke, C. J. 2011, MNRAS, 412, 13

Owen, J. E., Ercolano, B., \& Clarke, C. J. 2017, MNRAS, 472, 2955

Papaloizou, J., \& Terquem, C. 1999, ApJ, 521, 823

Pelletier, G., \& Pudritz, R. 1992, ApJ, 394, 117

Rapson, V. A., Pipher, J. L., Gutermuth, R. A., et al. 2014, ApJ, 794, 124

Rebull, L. M. 2001, AJ, 121, 1676

Rebull, L. M., Makidon, R. B., Strom, S. E., et al. 2002, AJ, 123, 1528

Rebull, L. M., Stauffer, J. R., Cody, A. M., et al. 2018, AJ, 155, 196

Ribas, Á., Merín, B., Bouy, H., et al. 2013, A\&A, 552, A115

Ribas, Á., Merín, B., Bouy, H., \& Maud, L. T. 2014, A\&A, 561, A54

Rieke, G. H., Young, E. T., Engelbracht, C. W., et al. 2004, ApJS, 154, 25

Robitaille, T. P. 2011, A\&A, 536, A79

Robitaille, T. P. 2017, A\&A, 600, A11

Robitaille, T. P., Whitney, B. A., Indebetouw, R., \& Wood, K. 2007, ApJS, 169, 328

Roquette, J., Bouvier, J., Alencar, S. H. P., Vaz, L. P. R., \& Guarcello, M. G. 2017, A\&A, 603, A106

Rosotti, G. P., Ercolano, B., Owen, J. E., \& Armitage, P. J. 2013, MNRAS, 430 1392

Safier, P. 1993, ApJ, 408, 115
Scally, A., \& Clarke, C. 2001, MNARS, 325, 449

Shu, F., Najita, J., Ostriker, E., et al. 1994, ApJ, 429, 781

Sousa, A. P., Alencar, S. H. P., Bouvier, J., et al. 2016, A\&A, 586, A47

Stauffer, J., Cody, A. M., Baglin, A., et al. 2014, AJ, 147, 83

Strom, K. M., Strom, S. E., Edwards, S., Cabrit, S., \& Skrutskie, M. F. 1989, AJ, 97,1451

Sung, H., Stauffer, J. R., \& Bessell, M. S. 2009, AJ, 138, 1116

Teixeira, P. S., Lada, C. J., Marengo, M., \& Lada, E. A. 2012, A\&A, 540, A83

van der Marel, N., Verhaar, B. W., van Terwisga, S., et al. 2016, A\&A, 592, A126

Vasconcelos, M. J., \& Bouvier, J. 2015, A\&A, 578, A89

Venuti, L., Bouvier, J., Flaccomio, E., et al. 2014, A\&A, 570, A82

Venuti, L., Bouvier, J., Irwin, J., et al. 2015, A\&A, 581, A66

Venuti, L., Bouvier, J., Cody, A. M., et al. 2017, A\&A, 599, A23

Walker, M. F. 1956, ApJS, 2, 365

Werner, M. W., Roellig, T. L., Low, F. J., et al. 2004, ApJS, 154, 1

White, R. J., \& Basri, G. 2003, ApJ, 582, 1109

Whitney, B. A., Indebetouw, R., Bjorkman, J. E., \& Wood, K. 2004, ApJ, 617, 1177

Wright, E. L., Eisenhardt, P. R. M., Mainzer, A. K., et al. 2010, AJ, 140, 1868

Zhu, Z., Nelson, R. P., Hartmann, L., Espaillat, C., \& Calvet, N. 2011, ApJ, 729, 47 


\section{Appendix A: Spectral energy distribution of all systems modeled with Hyperion model fitting}
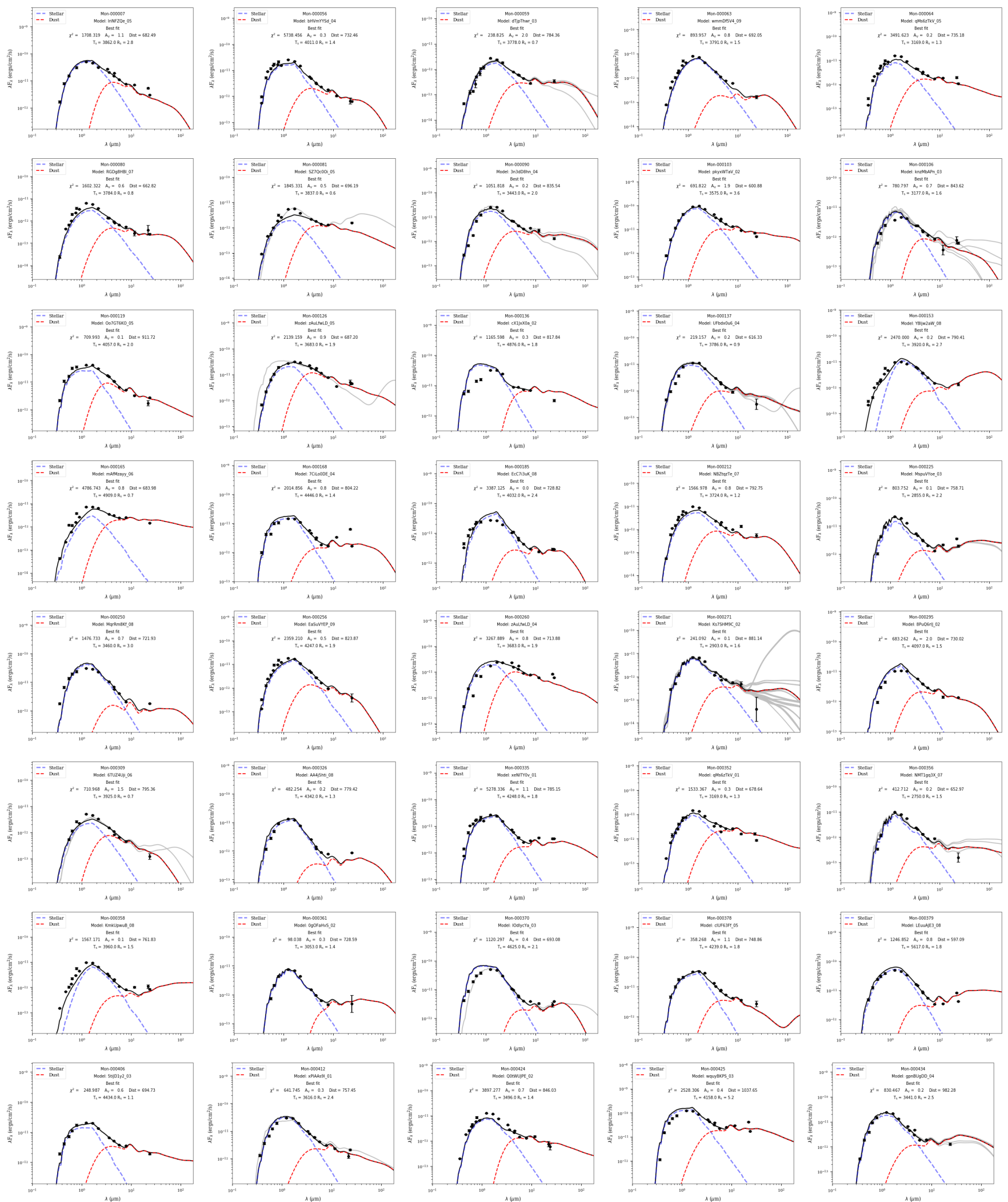

Fig. A.1. Spectral energy distribution of systems classified as full disks. The circles show data from the $U$ band $(0.3 \mu \mathrm{m})$ to the MIPS $24 \mu \mathrm{m}$ band. The black solid line is the best fit to the SED (Robitaille 2017) and the dashed lines correspond to the stellar (blue) and dust (red) emission components (Robitaille 2011). The gray solid lines are all the model with $\left(\chi^{2}-\chi_{\text {best }}^{2}\right)>3 n_{\text {data }}$, where $n_{\text {data }}$ is the number of available data points from the literature, following Robitaille (2017). All these systems were observed by Spitzer at $24 \mu \mathrm{m}$ and/or WISE at $22 \mu \mathrm{m}$. 
A. P. Sousa et al.: A study of accretion and disk diagnostics in the NGC 2264 cluster
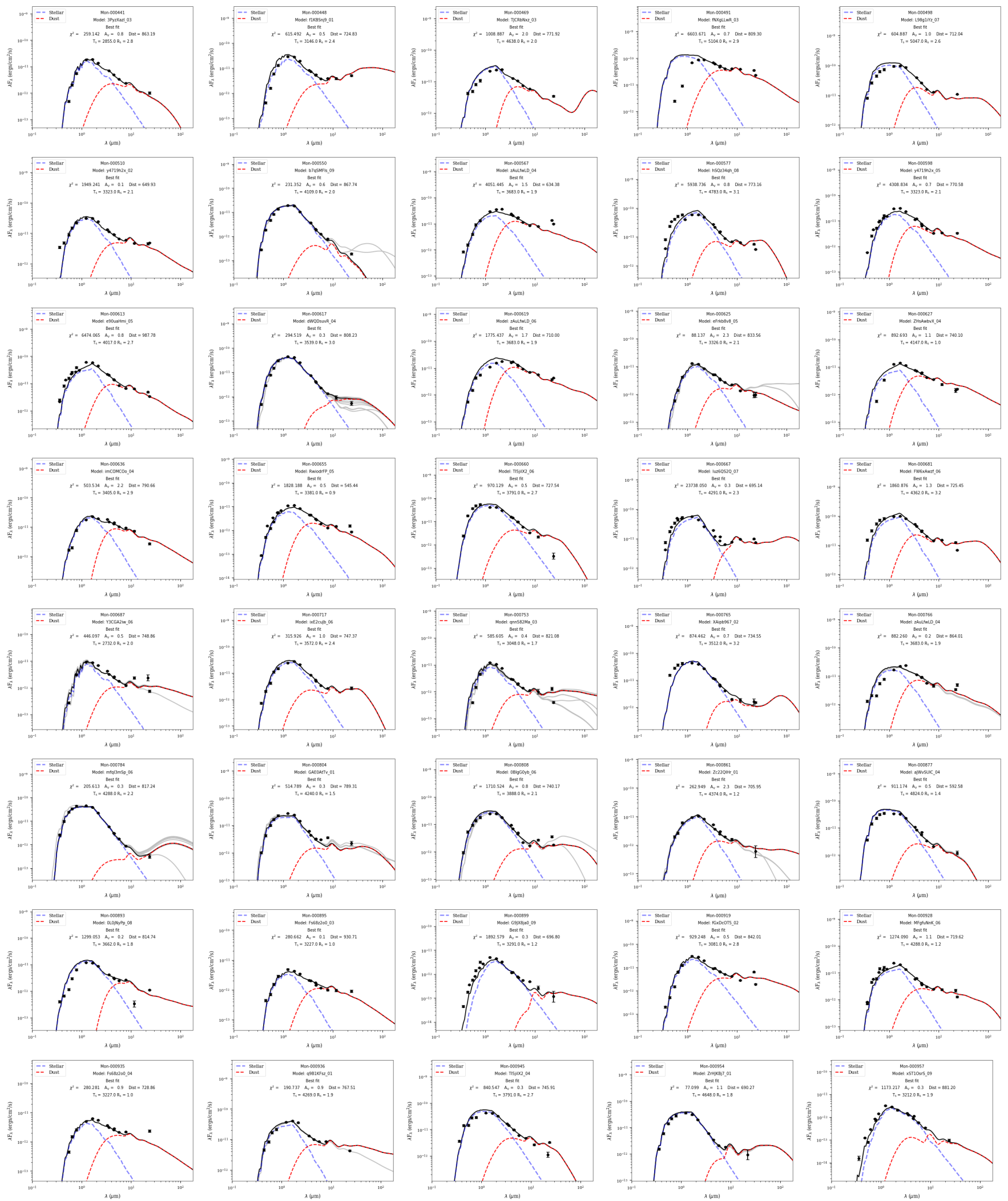

Fig. A.1. continued. 
A\&A 629, A67 (2019)
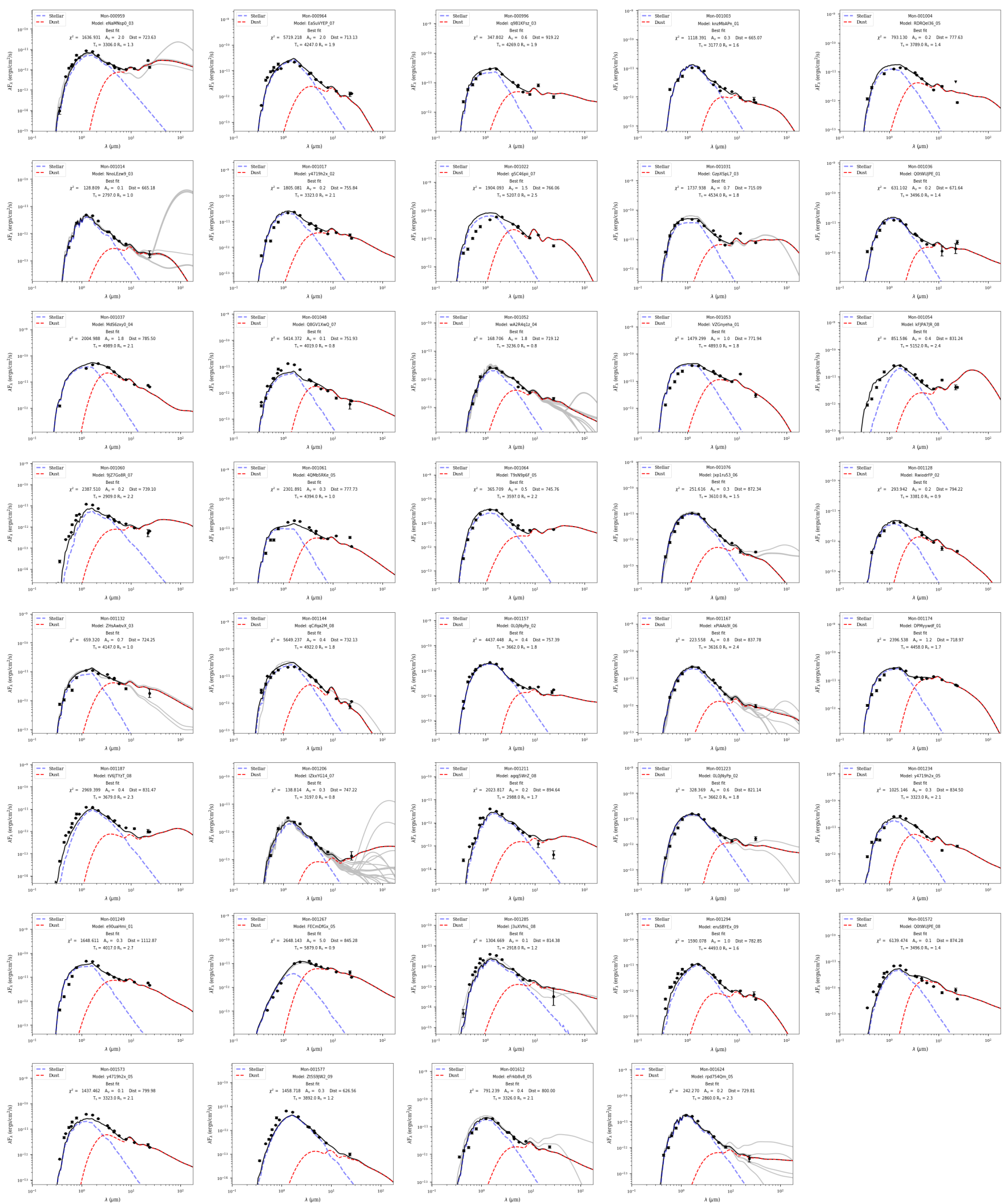

Fig. A.1. continued. 
A. P. Sousa et al.: A study of accretion and disk diagnostics in the NGC 2264 cluster
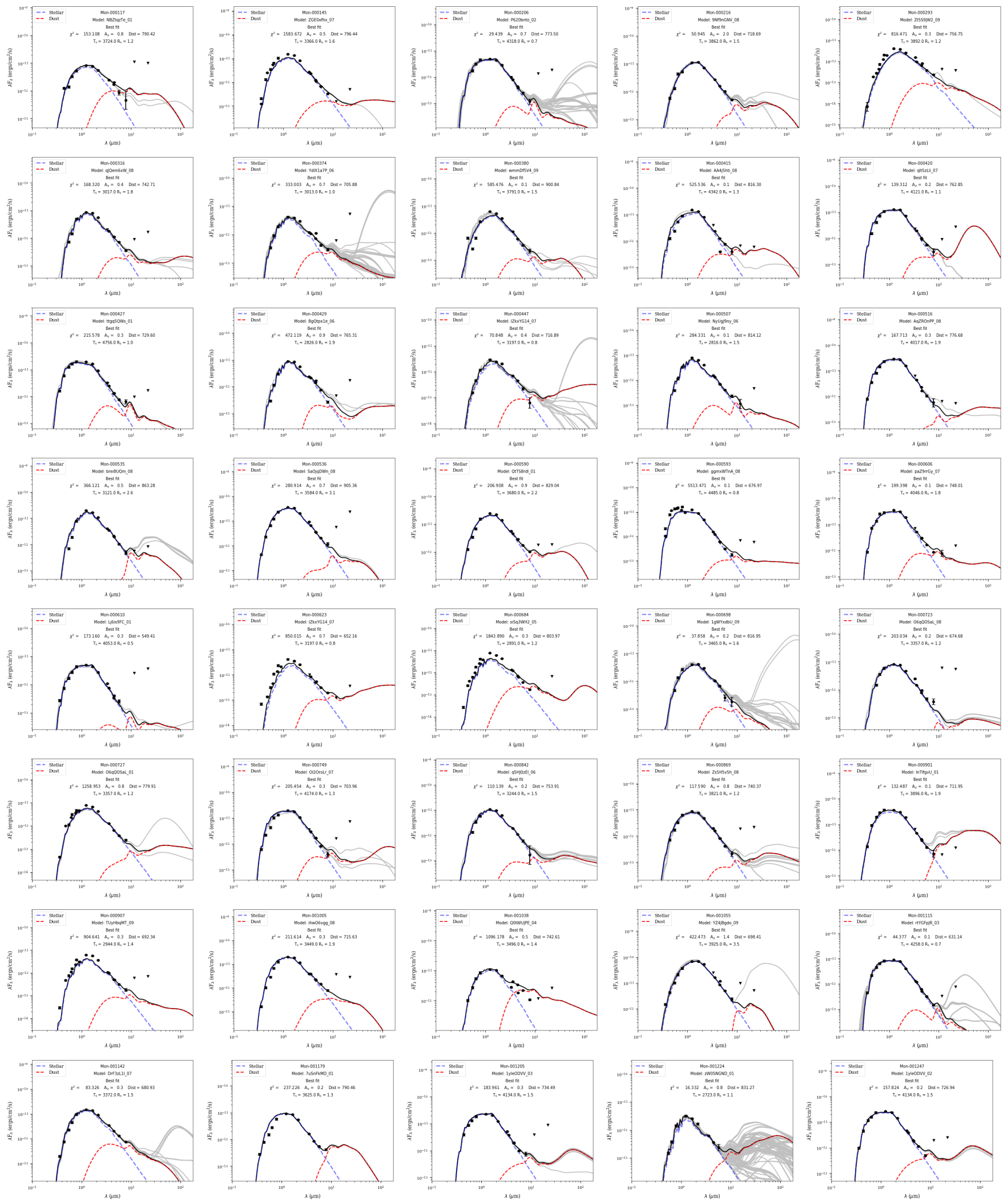

Fig. A.2. Same as Fig. A.1 but these systems were not observed by Spitzer at $24 \mu \mathrm{m}$ and/or WISE at $22 \mu \mathrm{m}$ or have only an upper limit of the flux at those wavelengths (upside down triangles). 

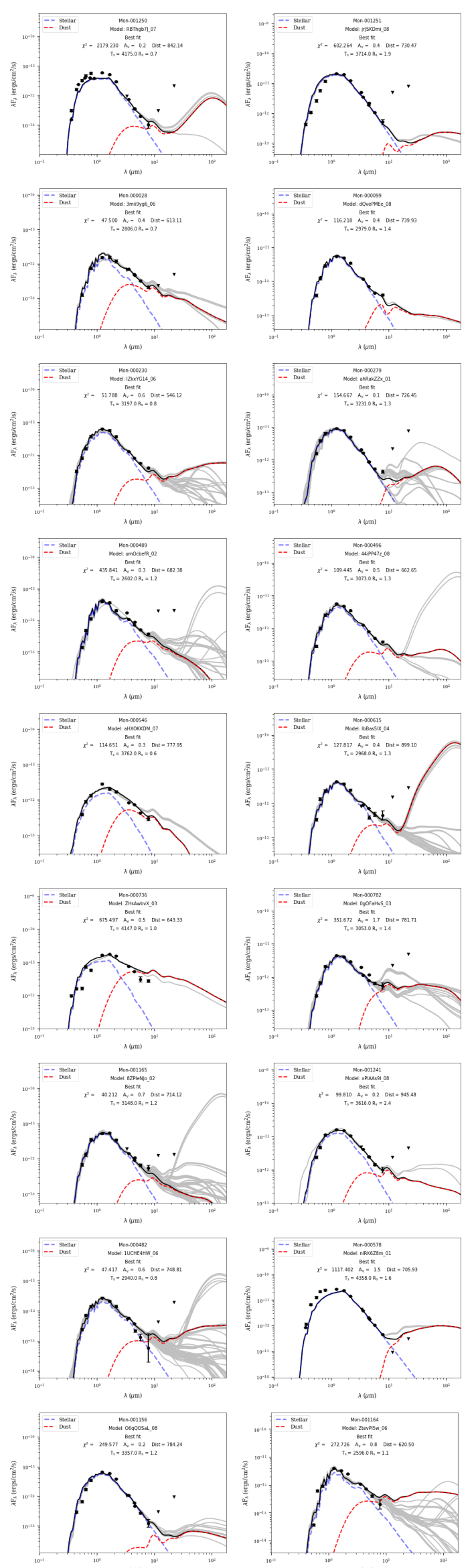
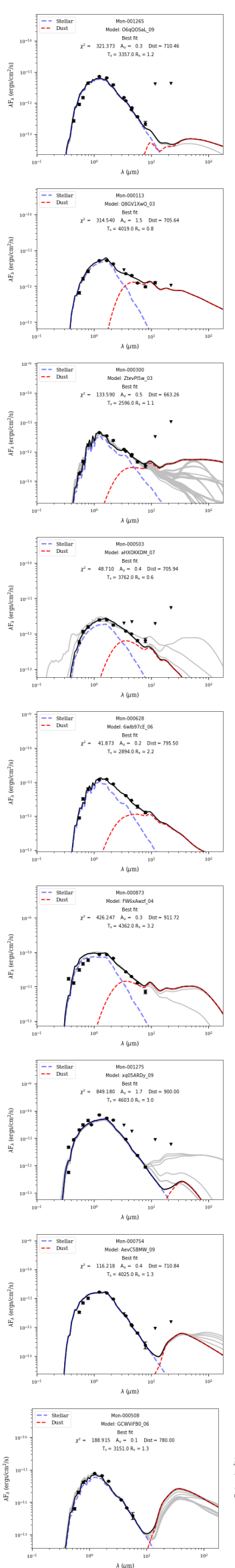
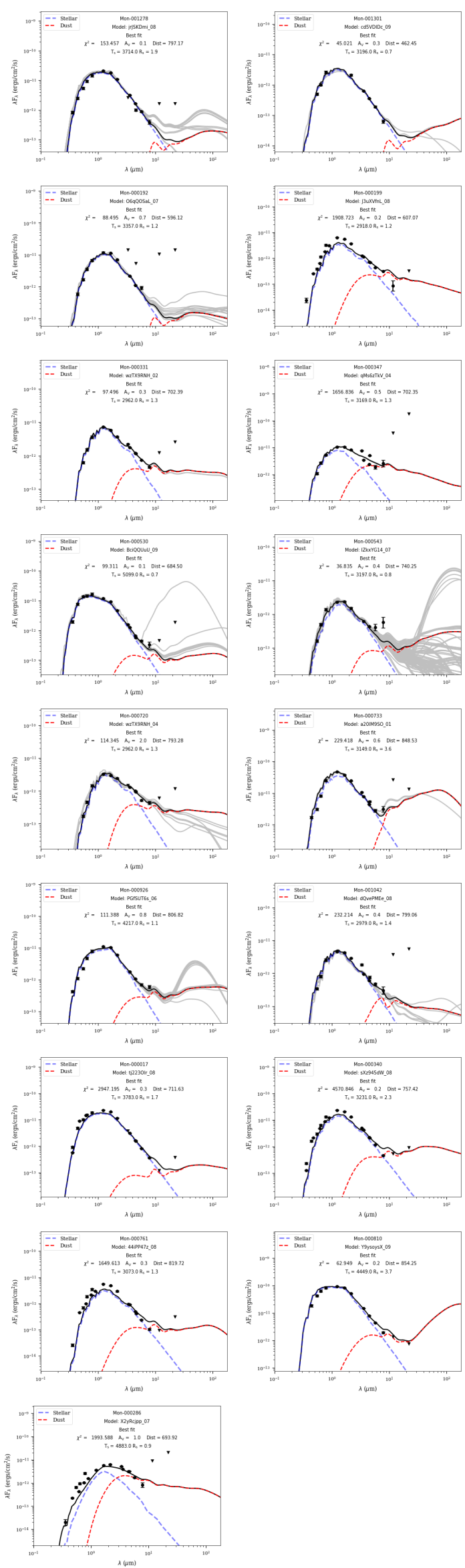

Fig. A.2. continued. 
A. P. Sousa et al.: A study of accretion and disk diagnostics in the NGC 2264 cluster
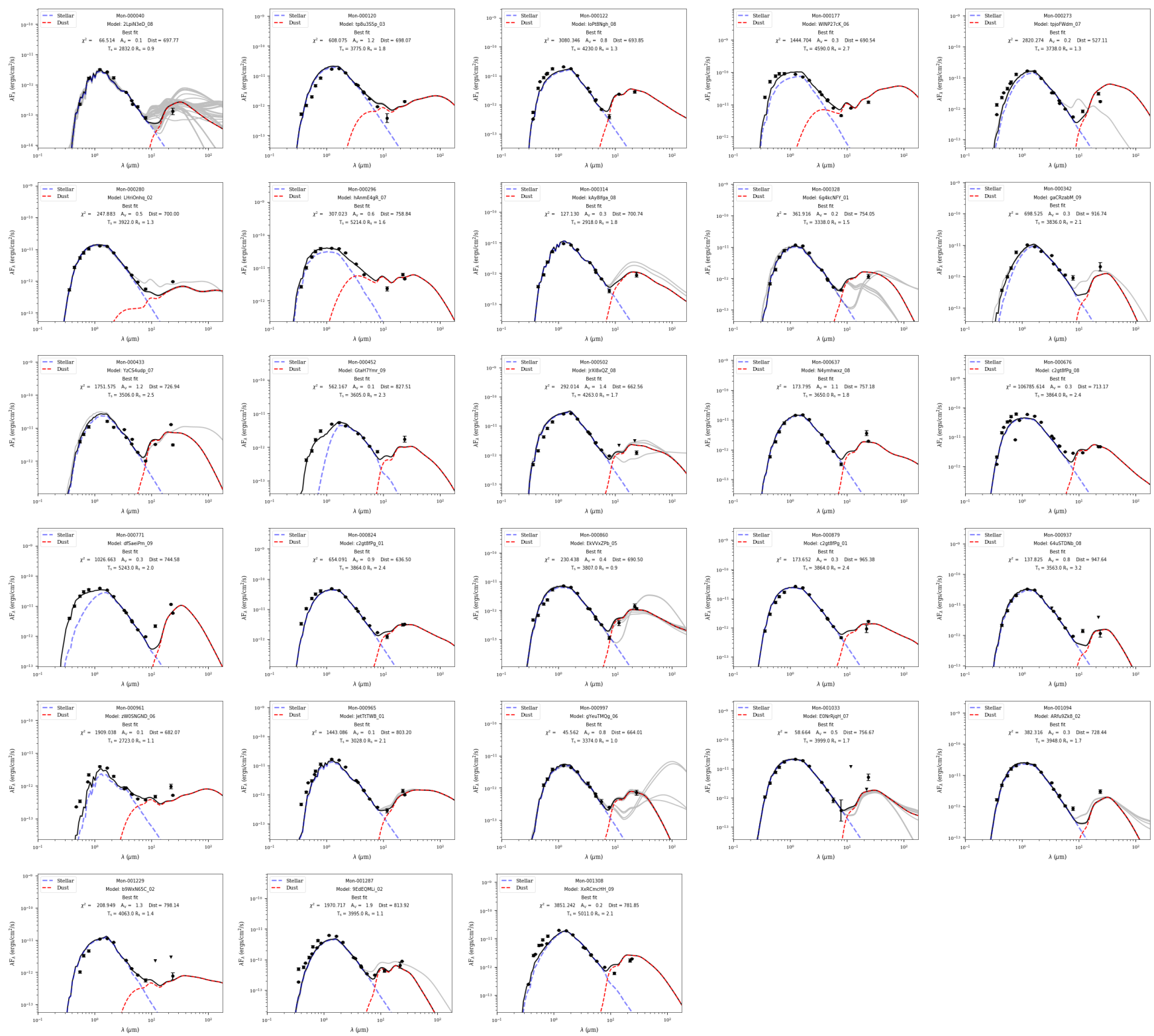

Fig. A.3. Same as Fig. A.1 but for systems classified as transition disk candidates. 

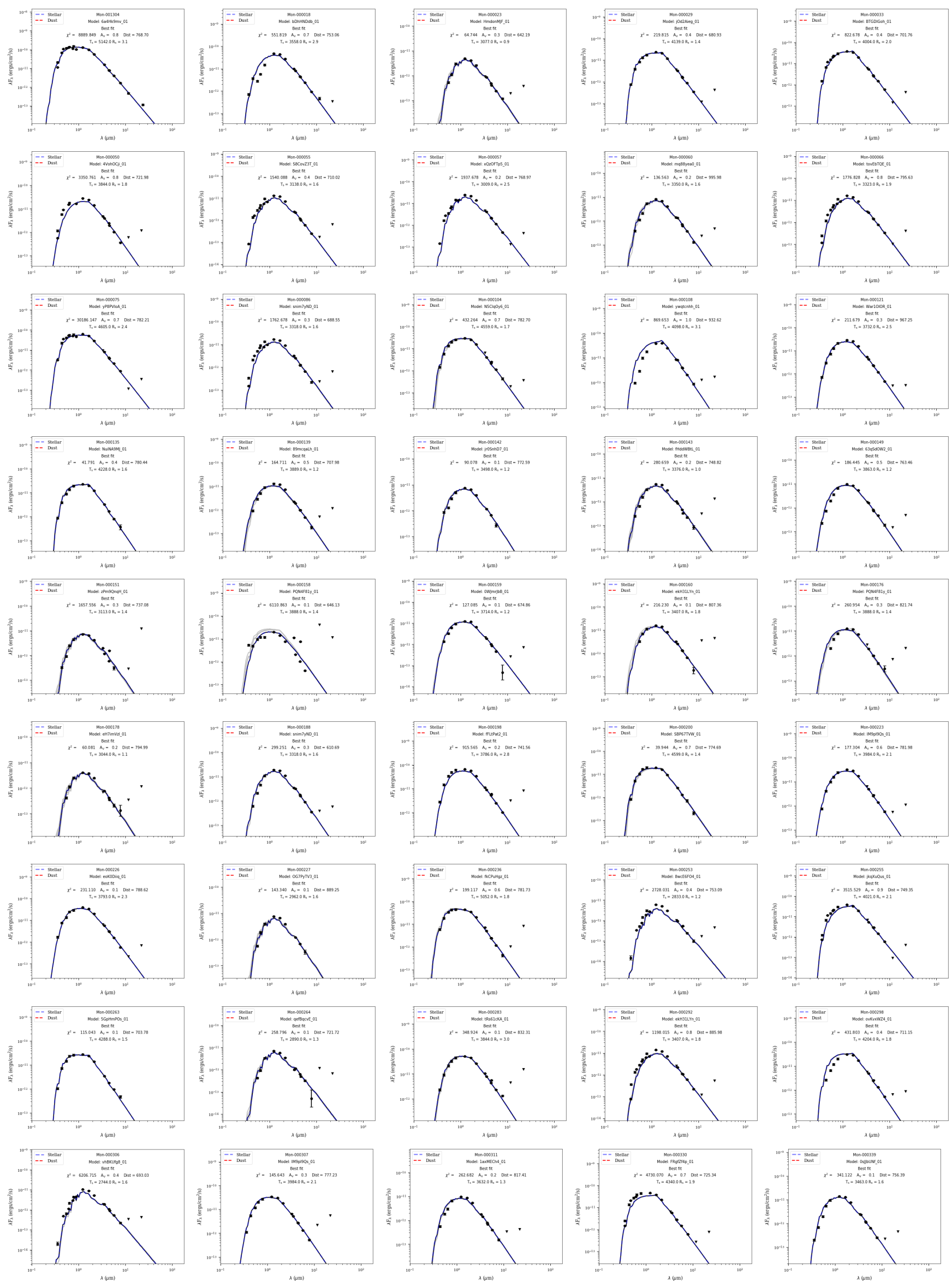

Fig. A.4. Same as Fig. A.1 but for stars classified as diskless. 
A. P. Sousa et al.: A study of accretion and disk diagnostics in the NGC 2264 cluster
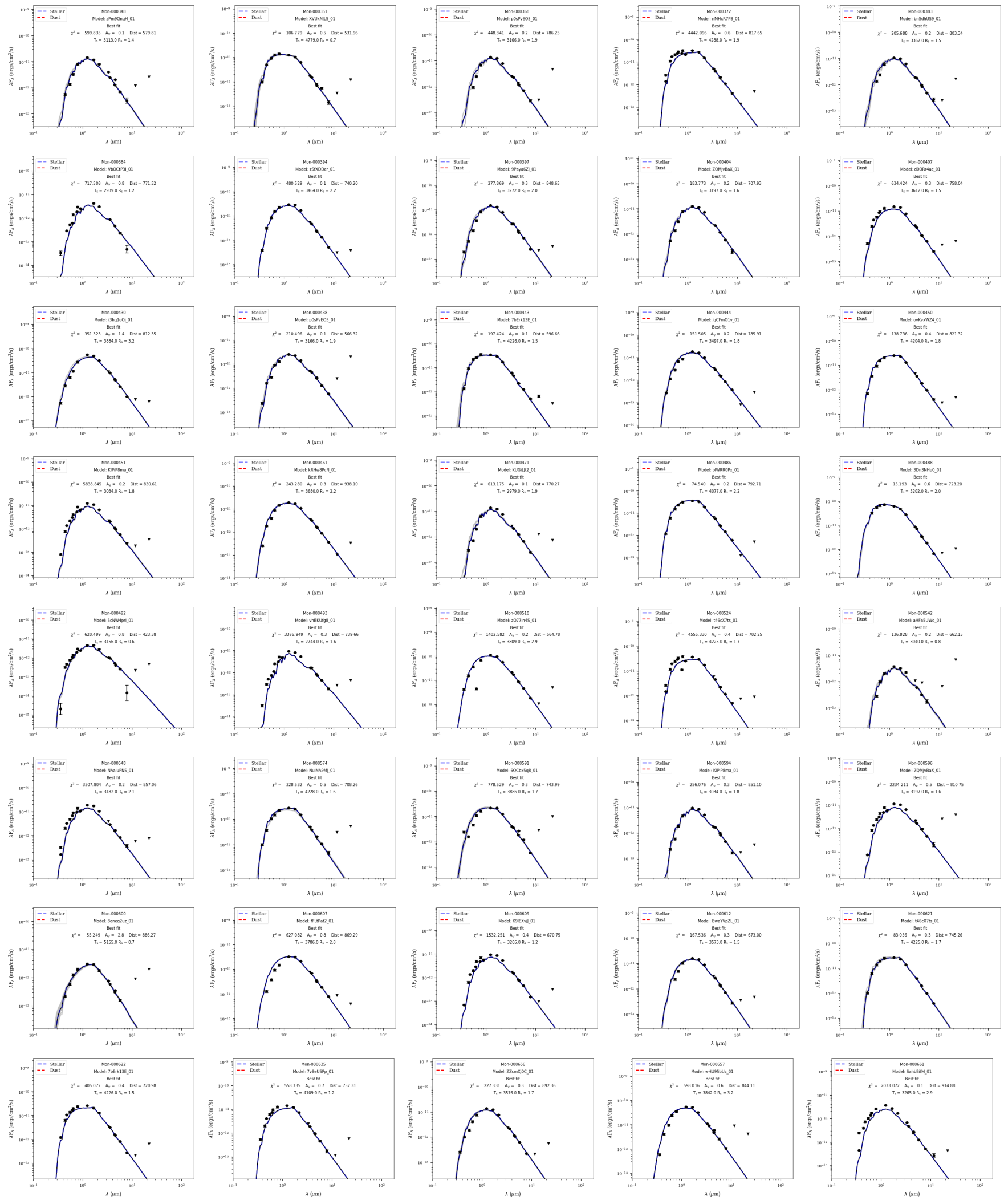

Fig. A.4. continued.

A67, page 25 of 27 
A\&A 629, A67 (2019)
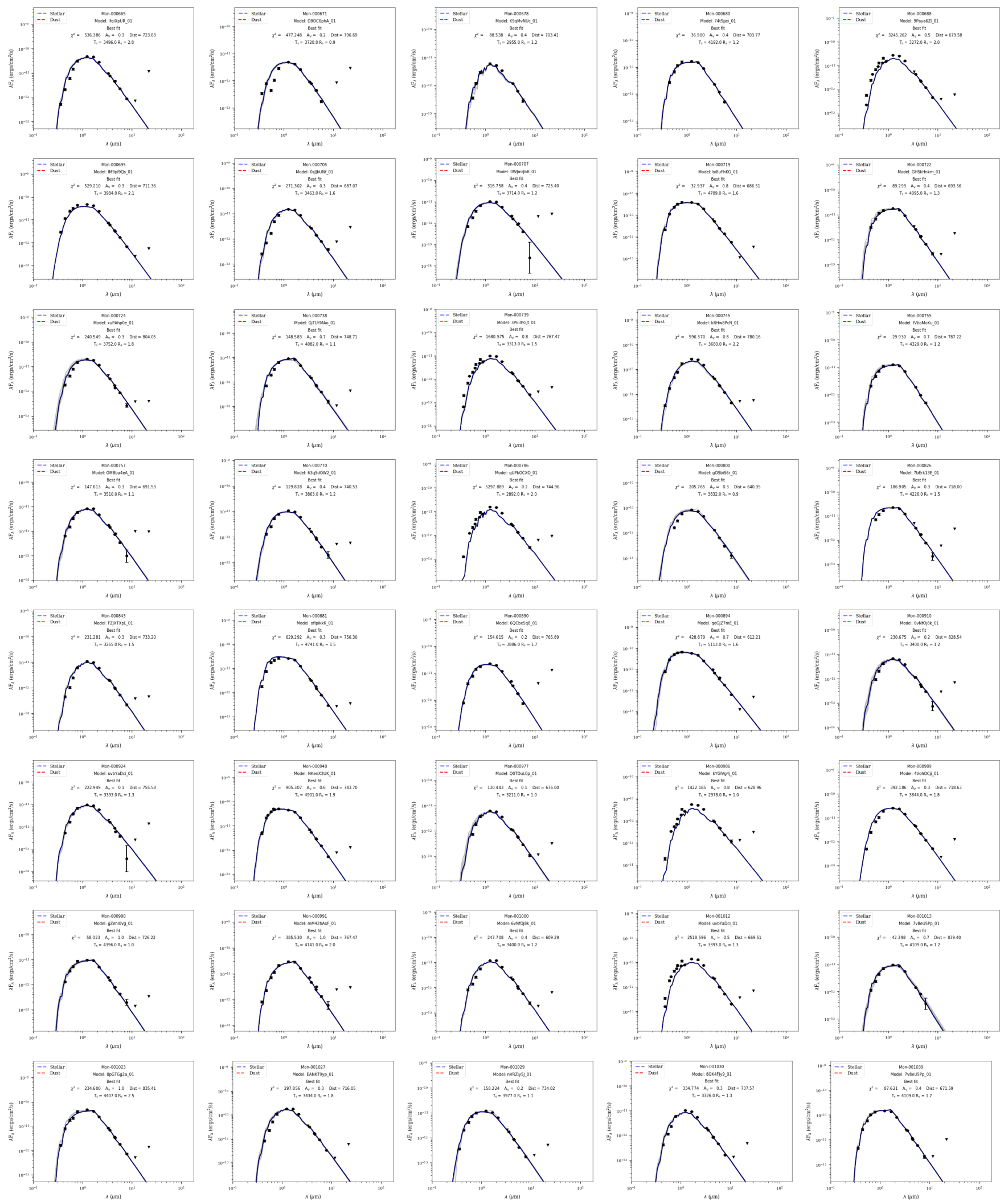

Fig. A.4. continued.

A67, page 26 of 27 
A. P. Sousa et al.: A study of accretion and disk diagnostics in the NGC 2264 cluster
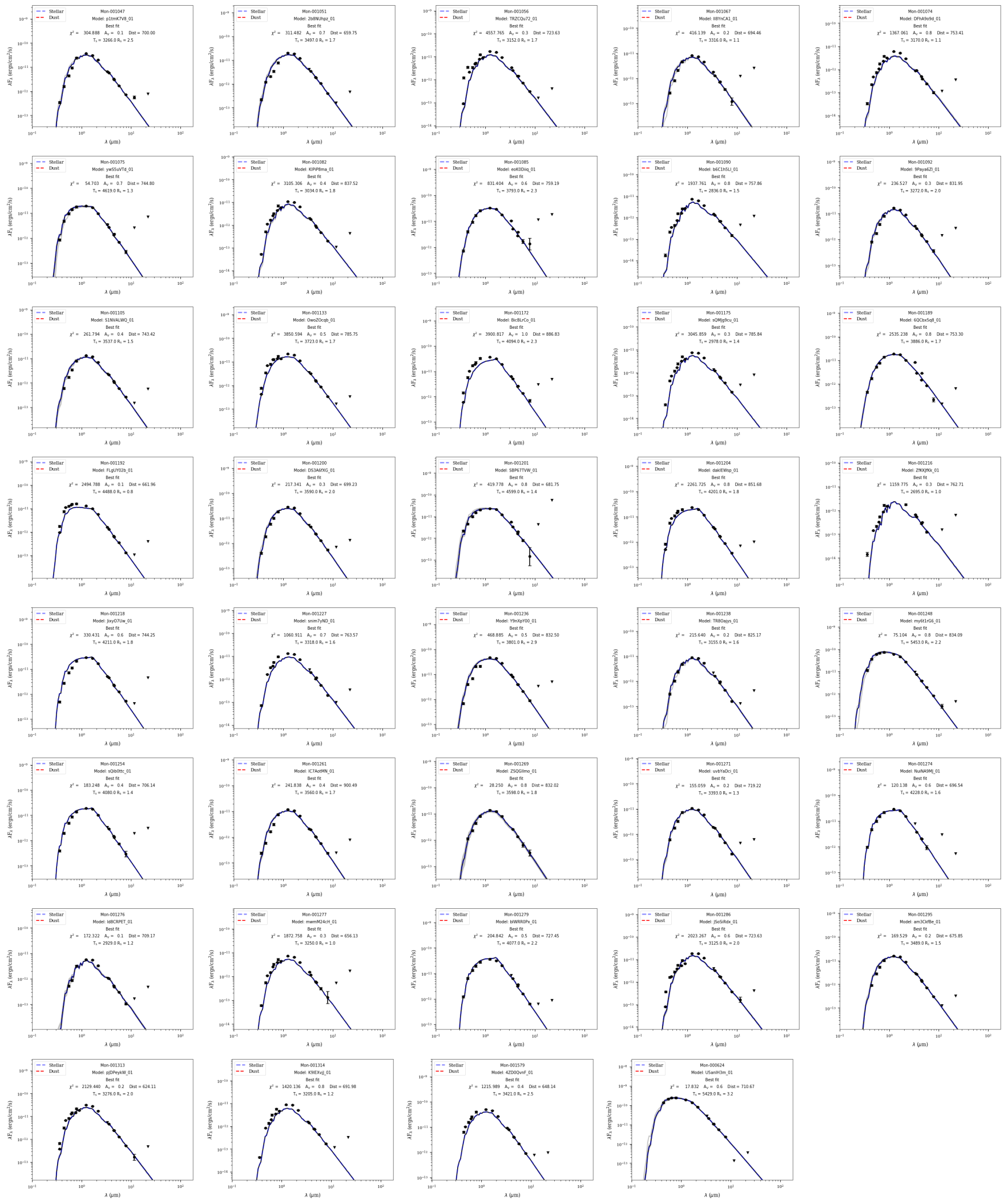

Fig. A.4. continued. 